\title{
Nonlinear Magnetoconvection in a Sparsely Packed Porous Medium
}

\author{
A. Benerji Babu, ${ }^{1}$ Ragoju Ravi, ${ }^{1}$ and S. G. Tagare ${ }^{2}$ \\ ${ }^{1}$ Department of Mathematics, National Institute of Technology Warangal, Warangal 506004, India \\ ${ }^{2}$ Disha Institute of Management and Technology, Satya Vihar, Vidhan Sabha-Chandrakhuri Marg, Raipur 492101, India
}

Correspondence should be addressed to A. Benerji Babu, benerji77@gmail.com

Received 2 July 2011; Revised 6 September 2011; Accepted 7 September 2011

Academic Editor: Steve Milan

Copyright ( $\odot 2011$ A. Benerji Babu et al. This is an open access article distributed under the Creative Commons Attribution License, which permits unrestricted use, distribution, and reproduction in any medium, provided the original work is properly cited.

Linear and weakly nonlinear properties of magnetoconvection in a sparsely packed porous medium are investigated. We have obtained the values of Takens-Bogdanov bifurcation points and codimension two bifurcation points by plotting graphs of neutral curves corresponding to stationary and oscillatory convection for different values of physical parameters relevant to magnetoconvection in a sparsely packed porous medium near a supercritical pitchfork bifurcation. We have derived a nonlinear two-dimensional Ginzburg-Landau equation with real coefficients by using Newell-Whitehead (1969) method. The effect of the parameter values on the stability mode is investigated and shown the occurrence of secondary instabilities namely, Eckhaus and Zigzag instabilities. We have studied Nessult number contribution at the onset of stationary convection. We have also derived two nonlinear one-dimensional coupled Ginzburg-Landau-type equations with complex coefficients near the onset of oscillatory convection at a supercritical Hopf bifurcation and discussed the stability regions of standing and travelling waves.

\section{Introduction}

Magnetoconvection in a porous medium uniformly heated from below is of considerable interest in geophysical fluid dynamics, as this phenomena may occur within the mushy layer of Earth's outer core. Earth's outer core consists of molten iron and lighter alloying element, sulphur in its molten form. This lighter alloying element present in the liquid phase is released as the new iron freezes due to supercooling onto the solid inner core. Hence we get mushy layer near the inner core boundary where the problem becomes convective instability in a porous medium [1]. The effect of geomagnetic field on the magnetoconvection instability is of interest in geophysics, particular in the study of Earth's interior where the molten liquid Iron is electrically conducting, which can become convectively unstable as a result of differential diffusion.

Magnetoconvection in an electrically conducting fluid in a nonporous medium has been studied extensively [2-8]. However, magnetoconvection in a porous medium has not received any attention inspite of its application in geophysical fluid dynamics problems. Palm et al., [9] investigated Rayleigh-Benard convection problem in a porous medium. Brand and Steinberg $[10,11]$ investigated convecting instabilities in binary liquid in a porous medium; However, Plam et al. [9] and Brand et al. have made use of Darcy's law $\left(-\nu \nabla^{2} \bar{V}\right.$ is replaced by $K \bar{V}$ where $K$ is the permeability of a porous medium. for nonporous medium $K$ is infinity). They have also not considered usual convective nonlinearity. It is well known that Darcy's law breaks down in situations where in other effects like viscous shear and inertia come into play. In fact Darcy's law is applicable to densely packed porous medium. An alternative to Darcy's equation is Brinkman equation and is of the form

$$
\nabla^{\prime} \rho^{\prime}-\rho^{\prime} \bar{g}=-\frac{\mu}{K} \bar{V}^{\prime}+\mu_{e} \nabla^{\prime 2} \bar{V}^{\prime},
$$

where $\mu$ is the fluid viscosity and $\mu_{e}$ is the effective fluid viscosity. Brinkman model is valid for a sparsely packed porous medium wherein there is more window fluid to flow 
so that the distortion of velocity give rise to the usual shear force. Lapwood [13] was the first to suggest the inclusion of convective term $\left(\bar{V}^{\prime} \cdot \nabla^{\prime}\right) \bar{V}^{\prime}$ in the momentum equation and study the Rayleigh-Benard convection in a sparsely packed porous medium. Recently, Tagare and Benerji [14] have investigated the problem of nonlinear convection in a sparsely packed porous medium due to thermal and compositional buoyancy.

In this paper we investigate the problem of magnetoconvection in a sparsely packed porous medium. The multiplicity of control parameters makes this system an interesting one for the study of hydrodynamic stability, bifurcation and turbulence [15]. Rudraiah [16] and Rudraiah and Vortmeyer [17] have studied both linear and steady nonlinear magnetoconvection in a sparsely packed porous medium using Brinkman model but they have taken effective viscosity $\mu_{e}$ same as fluid viscosity $\mu$. However, experiments show that the ratio of effective viscosity $\mu_{e}$ to fluid viscosity $\mu$ takes the value ranging from 0.5 to 10.9 [18]. In Section 2, we write basic dimensionless equations in Boussinesq approximation for magnetoconvection in a sparsely packed medium by using a momentum equation with effective viscosity different from fluid viscosity. In Section 3, we study linear stability analysis. In Section 4.1, by using multiple-scale analysis of Newell and Whitehead [19], we derive two-dimensional nonlinear Ginzburg-Landau equation in complex amplitude $A(X, Y, T)$ with real coefficients near the super critical pitchfork bifurcation. In Section 4.2, we show the occurrence of secondary instabilities such as Eckhaus instability and Zigzag instability. We have also considered the effect of Nusselt number on heat transport by magnetoconvection in a sparsely packed porous medium. In Section 5, we derive two nonlinear one-dimensional coupled Ginzburg-Landau type equations with complex coefficients near the onset of oscillatory convection at a supercritical Hopf bifurcation. Following Matthews and Rucklidge [20], we have dropped slow space dependence in $X$ and obtained two coupled ordinary differential equations in $A_{1 R}$ and $A_{1 L}$ and discussed the stability regions of travelling and standing waves. By obtaining a one-dimensional Ginzburg-Landau equation in complex amplitude $A(X, Y, T)$ with complex coefficients near a supercritical Hopf bifurcation, we have shown the condition for occurrence of Benjamin-Feir-type instability [21] for travelling and standing waves. In Section 6, we write conclusions of the paper.

\section{Basic Equations}

We consider an electrically and thermally conducting fluid saturating an infinite horizontal layer of a sparsely packed isotropic porous medium of depth $d$ with a uniform magnetic field $H_{0}$ in the vertical $z$-direction. This layer is heated from below, the upper and lower bounding surfaces of the layer are assumed to be stress-free. Physical properties of the fluid are assumed to be constant, except for the density in the buoyancy term, so that the Boussinesq approximation is valid. The temperature difference across the stress-free boundaries is $\Delta T^{\prime}$ and the flow in the sparsely packed porous medium is governed by the Darcy-LapwoodBrinkman model. The relevant basic equations are

$$
\begin{gathered}
\nabla^{\prime} \cdot \bar{V}^{\prime}=0, \quad \nabla^{\prime} \cdot \bar{H}^{\prime}=0 \\
\rho_{0}^{\prime}\left[\frac{1}{\phi} \frac{\partial \bar{V}^{\prime}}{\partial t^{\prime}}+\frac{1}{\phi^{2}}\left(\bar{V}^{\prime} \cdot \nabla^{\prime}\right) \bar{V}^{\prime}\right] \\
-\frac{\mu_{m}}{4 \pi}\left[H_{0}^{\prime} \frac{\partial \bar{H}^{\prime}}{\partial z^{\prime}}+\left(\bar{H}^{\prime} \cdot \nabla^{\prime}\right) \bar{H}^{\prime}\right] \\
=-\nabla\left(p^{\prime}+\frac{\mu_{m}}{8 \pi}\left|H^{\prime}\right|^{2}+\frac{\mu_{m} H_{0}}{4 \pi^{2}} H_{z}^{\prime}\right) \\
+\rho^{\prime} \bar{g}-\frac{\mu}{K} \bar{V}^{\prime}+\mu_{e} \nabla^{\prime 2} \bar{V}^{\prime} \\
M \frac{\partial T^{\prime}}{\partial t^{\prime}}+\left(\bar{V}^{\prime} \cdot \nabla^{\prime}\right) T^{\prime}=\kappa \nabla^{\prime 2} T^{\prime} \\
\phi \frac{\partial \bar{H}^{\prime}}{\partial t^{\prime}}=\nabla^{\prime} \times\left(\bar{V}^{\prime} \times H_{0}^{\prime} \hat{e}_{z}\right)+\nabla^{\prime} \times\left(\bar{V}^{\prime} \times \bar{H}^{\prime}\right)+\eta \nabla^{\prime 2} \bar{H}^{\prime} .
\end{gathered}
$$

The fluid density $\rho^{\prime}$ is described by

$$
\rho^{\prime}=\rho_{0}^{\prime}\left[1-\alpha\left(T^{\prime}-T_{b}^{\prime}\right)\right]
$$

where $\alpha=-\rho_{0}^{\prime-1}\left(\partial \rho^{\prime} / \partial T^{\prime}\right)$ is thermal expansion coefficient and $\rho_{0}^{\prime}$ is mean fluid density. Here $p^{\prime}$ is pressure, $\bar{V}^{\prime}$ is mean fluid velocity, $T^{\prime}$ is temperature, $\bar{H}^{\prime}$ is magnetic field, $\phi$ is porosity, $g$ is acceleration due to gravity, $K$ is permeability of porous medium, $\mu_{e}$ is coefficient of effective fluid viscosity, $\kappa$ is thermal diffusivity, $\mu_{m}$ is magnetic permeability, and $\eta$ is magnetic diffusivity. Equation (3) is known as DarcyLapwood-Brinkman equation and is valid for $0.8<\phi<1$. Givler and Altobelli [18] shown that the range of $\Lambda=\mu_{e} / \mu$ varies from 0.5 to $10.9 . M$ is dimensionless heat capacity and is defined as the ratio of the effective heat capacity of the porous medium to the heat capacity $\left(\rho^{\prime} C_{p}\right)_{f}$ of the fluid. In a nonporous medium, $\phi=M=\Lambda=1$ and $K \rightarrow \infty$ and (3) reduces to Navier-Stokes equation. In this paper, for sparsely packed porous medium, we consider $M=0.9, \phi=0.9$. The conduction state is characterized by

$$
\bar{V}_{s}^{\prime}=0, \quad T_{s}^{\prime}=T_{0}^{\prime}-\left(\frac{\Delta T^{\prime}}{d}\right) z^{\prime},
$$

and we take the temperature perturbation as $\theta^{\prime}=T^{\prime}-T_{s}^{\prime}$. We use the scaling

$$
\begin{gathered}
x=\frac{x^{\prime}}{d}, \quad y=\frac{y^{\prime}}{d}, \quad z=\frac{z^{\prime}}{d}, \quad t=\frac{t^{\prime}}{M d^{2} / \kappa}, \\
u=\frac{u^{\prime}}{\kappa / M d}, \quad v=\frac{v^{\prime}}{\kappa / M d}, \quad w=\frac{w^{\prime}}{\kappa / M d}, \\
\theta=\frac{\theta^{\prime}}{\Delta T^{\prime}}, \quad P=\frac{P^{\prime}}{\rho_{0}^{\prime} M^{-2} \kappa^{2} d^{-2}}, \quad \bar{H}=\frac{\bar{H}^{\prime}}{\kappa H_{0} / \eta} .
\end{gathered}
$$

Here $M d^{2} / \kappa$ is thermal diffusion time in a porous medium. Using (6) and (8), we can write basic dimensionless equations for magnetoconvection in a porous medium as

$$
\nabla \cdot \bar{V}=0, \quad \nabla \cdot \bar{H}=0,
$$




$$
\begin{gathered}
\frac{1}{M^{2} \phi \operatorname{Pr}_{1}}\left[\frac{\partial \bar{V}}{\partial t}+\frac{1}{\phi}(\bar{V} \cdot \nabla) \bar{V}\right]-Q \frac{\operatorname{Pr}_{2}}{\operatorname{Pr}_{1}}(\bar{H} \cdot \nabla) \bar{H}-Q \frac{\partial \bar{H}}{\partial z} \\
=-\nabla\left(\frac{P}{M \operatorname{Pr}_{1}}+\frac{Q}{2} \frac{\operatorname{Pr}_{2}}{\operatorname{Pr}_{1}}|\bar{H}|^{2}+Q H_{z}\right)-\frac{1}{M D_{a}} \bar{V} \\
+\frac{\Lambda}{M} \nabla^{2} \bar{V}+R \theta \hat{e}_{z}, \\
\quad \frac{\partial \theta}{\partial t}+\frac{1}{M}(\bar{V} \cdot \nabla) \theta=\frac{w}{M}+\nabla^{2} \theta \\
\phi \frac{\operatorname{Pr}_{2}}{\operatorname{Pr}_{1}} \frac{\partial \bar{H}}{\partial t}-M \nabla^{2} \bar{H}=\nabla \times\left(\bar{V} \times \hat{e}_{z}\right)+\frac{\operatorname{Pr}_{2}}{\operatorname{Pr}_{1}} \nabla \times(\bar{V} \times \bar{H}) .
\end{gathered}
$$

The dimensionless parameters required for the description of the motion are Rayleigh number $R=g \alpha \Delta T d^{3} / \kappa \nu$, thermal Prandtl number $\operatorname{Pr}_{1}=\nu / \kappa$, magnetic Prandtl number $\operatorname{Pr}_{2}=$ $\nu / \eta$, Chandrasekhar number $Q=\mu_{m} H_{0}^{2} d^{2} / 4 \pi \rho_{0} \nu \eta$, and Darcy number $D_{a}=\kappa / d^{2}$. The Curl of (10) gives

$$
\begin{gathered}
\left(\frac{1}{M^{2} \phi \operatorname{Pr}_{1}} \frac{\partial}{\partial t}+\frac{1}{M D_{a}}-\frac{\Lambda}{M} \nabla^{2}\right) \bar{\omega}-Q \frac{\partial \bar{J}}{\partial z}-R\left(\nabla \times\left(\theta \widehat{e}_{z}\right)\right) \\
=Q \frac{\operatorname{Pr}_{2}}{\operatorname{Pr}_{1}}[\nabla \times(\bar{H} \cdot \nabla) \bar{H}] \\
\quad-\frac{1}{M^{2} \phi^{2} \operatorname{Pr}_{1}}[\nabla \times(\bar{V} \cdot \nabla) \bar{V}]
\end{gathered}
$$

where vorticity $\bar{\omega}=\nabla \times \bar{V}$, current $\bar{J}=\nabla \times \bar{H}$ and

$$
\begin{aligned}
& \nabla \times[(\bar{V} \cdot \nabla) \bar{V}]=[(\bar{V} \cdot \nabla) \bar{\omega}-(\bar{\omega} \cdot \nabla) \bar{V}], \\
& \nabla \times[(\bar{H} \cdot \nabla) \bar{H}]=[(\bar{H} \cdot \nabla) \bar{J}-(\bar{J} \cdot \nabla) \bar{H}] .
\end{aligned}
$$

The Curl of (13) in turn gives, after use of (9),

$$
\begin{aligned}
\left(\frac{1}{M^{2} \phi \operatorname{Pr}_{1}} \frac{\partial}{\partial t}+\frac{1}{M D_{a}}-\frac{\Lambda}{M} \nabla^{2}\right) \nabla^{2} \bar{V} \\
-R\left[\left(\nabla^{2} \theta\right) \hat{e}_{z}-\nabla\left(\frac{\partial \theta}{\partial z}\right)\right]-Q \frac{\partial}{\partial z}\left(\nabla^{2} \bar{H}\right) \\
=\frac{1}{M^{2} \phi^{2} \operatorname{Pr}_{1}} \nabla \times[(\bar{V} \cdot \nabla) \bar{\omega}-(\bar{\omega} \cdot \nabla) \bar{V}] \\
-Q \frac{\operatorname{Pr}_{2}}{\operatorname{Pr}_{1}} \nabla \times[(\bar{H} \cdot \nabla) \bar{J}-(\bar{J} \cdot \nabla) \bar{H}] .
\end{aligned}
$$

Now taking the scalar product of (12), (13), and (15) with $\hat{e}_{z}$, we get,

$$
\left(\phi \frac{\operatorname{Pr}_{2}}{\operatorname{Pr}_{1}} \frac{\partial}{\partial t}-M \nabla^{2}\right) H_{z}-\frac{\partial w}{\partial z}=\frac{\operatorname{Pr}_{2}}{\operatorname{Pr}_{1}} \hat{e}_{z} \cdot[\nabla \times(\bar{V} \times \bar{H})]
$$

$$
\begin{gathered}
\left(\frac{1}{M^{2} \phi \operatorname{Pr}_{1}} \frac{\partial}{\partial t}+\frac{1}{M D_{a}}-\frac{\Lambda}{M} \nabla^{2}\right) \nabla^{2} \bar{\omega}_{z}+Q \frac{\partial J_{z}}{\partial z}-R \frac{\partial \theta}{\partial x} \\
=Q \frac{\operatorname{Pr}_{2}}{\operatorname{Pr}_{1}} \hat{e}_{z} \cdot \nabla \times(\bar{H} \cdot \nabla) \bar{H}-\frac{1}{M^{2} \phi^{2} \operatorname{Pr}_{1}} \hat{e}_{z} \\
\cdot[\nabla \times(\bar{V} \cdot \nabla) \bar{V}], \\
\left(\frac{1}{M^{2} \phi \operatorname{Pr}_{1}} \frac{\partial}{\partial t}+\frac{1}{M D_{a}}-\frac{\Lambda}{M} \nabla^{2}\right) \nabla^{2} w-R \nabla_{h}^{2} \theta-Q \frac{\partial}{\partial z}\left(\nabla^{2} H_{z}\right) \\
=\frac{1}{M^{2} \phi^{2} \operatorname{Pr}_{1}} \hat{e}_{z} \cdot[\nabla \times[(\bar{V} \cdot \nabla) \bar{\omega}-(\bar{\omega} \cdot \nabla) \bar{V}]] \\
-Q \frac{\operatorname{Pr}_{2}}{\operatorname{Pr}_{1}} \hat{e}_{z} \cdot[\nabla \times[(\bar{H} \cdot \nabla) \bar{J}-(\bar{J} \cdot \nabla) \bar{H}]]
\end{gathered}
$$

Geophysically acceptable velocities of propagating instabilities corresponding to geometric scalar variations occur only $\mathrm{Pr}_{2} / \mathrm{Pr}_{1}>1$ (where instabilities develop in ohmic diffusion timescale $\left.d^{2} / \eta\right), \operatorname{Pr}_{2} / \operatorname{Pr}_{1}=2$ and 5, when the turbulent is present in the Earth's outer core. In the case of $\operatorname{Pr}_{2} / \operatorname{Pr}_{1} \ll 1$ the instabilities are extremely slow depending on the thermal diffusion timescale $d^{2} / \kappa$. Using (11), (18), and (16) can be reduced to a form

$$
\mathcal{L} w=\mathcal{N}
$$

where

$$
\begin{gathered}
\mathcal{L}=\left(\mathscr{D}_{\phi} \mathscr{D}_{\mathrm{Pr}_{1}}-Q \frac{\partial^{2}}{\partial z^{2}}\right) \mathscr{D} \nabla^{2}-\frac{R}{M} \nabla_{h}^{2} \mathcal{D}_{\phi}, \\
\mathcal{N}=Q \mathscr{D} \nabla^{2} \frac{\operatorname{Pr}_{2}}{\operatorname{Pr}_{1}} \frac{\partial}{\partial z}\left[(\bar{H} \cdot \nabla) w-(\bar{V} \cdot \nabla) H_{z}\right] \\
+\mathscr{D} \mathscr{D}_{\phi} \hat{e}_{z} \cdot\left[\left[\frac{1}{M^{2} \phi^{2} \operatorname{Pr}_{1}} \nabla \times[(\bar{V} \cdot \nabla) \bar{\omega}-(\bar{\omega} \cdot \nabla) \bar{V}]\right]\right. \\
\left.-\left[Q \frac{\operatorname{Pr}_{2}}{\operatorname{Pr}_{1}} \nabla \times[(\bar{H} \cdot \nabla) \bar{J}-(\bar{J} \cdot \nabla) \bar{H}]\right]\right] \\
-\frac{R}{M} \nabla_{h}^{2} \mathscr{D}_{\phi}(\bar{V} \cdot \nabla) \theta
\end{gathered}
$$

here

$$
\begin{gathered}
\mathscr{D}=\left(\frac{\partial}{\partial t}-\nabla^{2}\right), \quad \mathscr{D}_{\phi}=\left(\phi \frac{\operatorname{Pr}_{2}}{\operatorname{Pr}_{1}} \frac{\partial}{\partial t}-M \nabla^{2}\right) \\
\mathscr{D}_{\operatorname{Pr}_{1}}=\left(\frac{1}{M^{2} \phi \operatorname{Pr}_{1}} \frac{\partial}{\partial t}+\frac{1}{M D_{a}}-\frac{\Lambda}{M} \nabla^{2}\right) \\
\nabla_{h}^{2}=\frac{\partial^{2}}{\partial x^{2}}, \quad \nabla^{2}=\left(\frac{\partial^{2}}{\partial x^{2}}+\frac{\partial^{2}}{\partial z^{2}}\right) .
\end{gathered}
$$


Boundary Conditions. We assume that fluid is contained between $z=0$ and $z=1$, where $z=0$ corresponds to boundary of solid iron core with Earth's mushy layer and $z=1$ corresponds to boundary of Earth's mushy layer with Earth's outer liquid core. For perfectly conducting boundary with temperature, we have

$$
\theta=0, \quad H_{z}=0 \quad \text { on } z=0, z=1 \forall x, y .
$$

Also the normal component of the velocity would vanish on $z=0, z=1$, that is,

$$
w=0 \quad \text { on } z=0, z=1 \forall x, y .
$$

However, there are two more conditions to be imposed on velocity depending on the nature of the surface. In this paper we consider free-free boundary conditions, that is, on surfaces the tangential stresses vanish, which is equivalent to

$$
\begin{aligned}
& P_{x z}=\mu\left(\frac{\partial u}{\partial z}+\frac{\partial w}{\partial x}\right)=0 \\
& P_{y z}=\mu\left(\frac{\partial v}{\partial z}+\frac{\partial w}{\partial y}\right)=0
\end{aligned}
$$

where $\mu=\gamma \rho_{0}$ is dynamic viscosity. Since $w$ vanishes for $x, y$ on $z=0, z=1$, it follows that $\partial u / \partial z=\partial v / \partial z=0$ on a free surface $z=0, z=1$. Hence from equation of continuity we have $\partial^{2} w / \partial z^{2}=0$ on $z=0, z=1$ for all $x, y$. In this paper we have considered only the idealized stress-free conditions on the surface and vanishing of temperature fluctuations. Thus $w=D^{2} w=D^{4} w=0$ at $z=0,1 . w$ and its even derivatives vanish at $z=0$ and $z=1$.

\section{Linear Stability Analysis}

We perform a linear stability analysis of the problem by substituting

$$
w=W(z) e^{i q x+p t},
$$

into linearized version of (19) is $\mathcal{L} w=0$, and obtaining an equation

$$
\begin{gathered}
{\left[\left(D^{2}-q^{2}-p\right)\left(M D^{2}-M q^{2}-p \phi \frac{\operatorname{Pr}_{2}}{\operatorname{Pr}_{1}}\right)\left(D^{2}-q^{2}\right)\right.} \\
\left.\times\left\{\frac{\Lambda}{M}\left(D^{2}-q^{2}\right)-\frac{1}{M D_{a}}-\frac{p}{M^{2} \phi \operatorname{Pr}_{1}}\right\}\right] W \\
=\left[-\frac{R q^{2}}{M}\left(M D^{2}-M q^{2}-p \phi \frac{\operatorname{Pr}_{2}}{\operatorname{Pr}_{1}}\right)\right. \\
\left.+Q\left(D^{2}-q^{2}\right)\left(D^{2}-q^{2}-p\right) D^{2}\right] W .
\end{gathered}
$$

We consider stress-free boundary conditions, then $W=$ $D^{2} W=0$ on $z=0, z=1$ for all $x, y$. Thus we can assume $W=\sin \pi z$.

Substituting $W=\sin \pi z$ and $p=i \omega$ into (27), we get

$$
R=\frac{M}{q^{2}}\left[A_{1}+i \omega\left(A_{2} \omega^{2}+A_{3}\right)\right],
$$

$$
\begin{aligned}
& A_{1}=\mathcal{K}[\{ M \delta^{6}\left(\frac{1}{D_{a}}+\Lambda \delta^{2}\right)+M \delta^{4} \\
&\left.\times\left(Q \pi^{2}-\omega^{2} \frac{\operatorname{Pr}_{2}}{M^{2} \operatorname{Pr}_{1}^{2}}-\frac{\omega^{2}}{M \phi \operatorname{Pr}_{1}}-\omega^{2} \phi \frac{\Lambda}{M} \frac{\operatorname{Pr}_{2}}{\operatorname{Pr}_{1}}\right)\right\} \\
&+ \omega^{2} \frac{\operatorname{Pr}_{2}}{\operatorname{Pr}_{1}}\left\{\frac{\operatorname{Pr}_{2}}{\operatorname{Pr}_{1}}\left(\delta^{4} \phi^{2} \frac{\Lambda}{M}-\frac{\omega^{2}}{M \phi \operatorname{Pr}_{1}^{2}}\right)\right. \\
& \times \delta^{4}\left(\Lambda \phi+\frac{1}{M \operatorname{Pr}_{1}}\right)+Q \pi^{2} \phi \\
&\left.\left.+\frac{\delta \phi^{2} \operatorname{Pr}_{2}}{M D_{a} \operatorname{Pr}_{1}}\right\}\right], \\
& A_{2}=\mathcal{K}\left[\delta^{2}\left(\frac{\operatorname{Pr}_{2}}{\operatorname{Pr}_{1}}\right)^{2}\left(\phi^{2} \frac{\Lambda}{M}+\frac{\phi}{M^{2} \operatorname{Pr}_{1}}\right)+\frac{\phi^{2} \operatorname{Pr}_{2}^{2}}{M D_{a} \operatorname{Pr}_{1}^{2}}\right], \\
& A_{3}=\mathcal{K}\left[\delta^{4}\left(M \Lambda+\frac{1}{\phi \operatorname{Pr}_{1}}\right)+\frac{M \delta^{2}}{D_{a}}+Q \pi^{2}\left(M-\phi \frac{\operatorname{Pr}_{2}}{\operatorname{Pr}_{1}}\right)\right],
\end{aligned}
$$

where $\mathcal{K}=\delta^{2}\left(M^{4} \delta^{4}+\omega^{2} \phi^{2} \operatorname{Pr}_{2}^{2} / \operatorname{Pr}_{1}^{2}\right)^{-1}$ and $\delta^{2}=\left(\pi^{2}+q^{2}\right)$, from relation equation (30), $A_{2}>0$.

3.1. Stationary Convection ( $\omega=0$ ). Substituting $\omega=0$ in (28), we get

$$
R_{s}=\frac{\delta_{s}^{2}}{q_{s}^{2}}\left[\delta_{s}^{2}\left(\frac{1}{D_{a}}+\delta_{s}^{2} \Lambda\right)+Q \pi^{2}\right]
$$

here $R_{s}$ is the value of the Rayleigh number for stationary convection. The minimum value of $R_{s}$ is obtained for $q_{s}=$ $q_{\mathrm{sc}}$. where

$$
2 \Lambda\left(\frac{q}{\pi}\right)^{6}+\left(3 \Lambda+\frac{1}{\pi^{2} D D_{a}}\right)\left(\frac{q}{\pi}\right)^{4}=\Lambda+\frac{Q}{\pi^{2}}+\frac{1}{\pi^{2} D D_{a}} .
$$

The wave number is identical to that for the single component fluid, while the threshold for the onset of stationary convection at pitchfork bifurcation is given by (34) with $q_{s}=q_{\mathrm{sc}}$,

$$
R_{\mathrm{sc}}=\frac{\delta_{\mathrm{sc}}^{2}}{q_{\mathrm{sc}}^{2}}\left[\delta_{\mathrm{sc}}^{2}\left(\frac{1}{D_{a}}+\delta_{\mathrm{sc}}^{2} \Lambda\right)+Q \pi^{2}\right]
$$

where $\delta_{\mathrm{sc}}^{2}=\pi^{2}+q_{\mathrm{sc}}^{2}$. Thus the magnetic field inhibits the onset of stationary convection.

3.2. Oscillatory Convection $\left(\omega^{2}>0\right)$. For the oscillatory convection $(\omega \neq 0)$ and from (28), $R$ will be complex. But the physical meaning of $R$ requires it to be real. The condition that $R$ is real implies that imaginary part of (28) is zero, that is,

$$
A_{2} \omega^{2}+A_{3}=0
$$


where $A_{2}$ and $A_{3}$ are given by (30) and (31). For oscillatory convection $\omega^{2}=-A_{3} / A_{2}>0$ since $A_{2}>0$, for oscillatory convection $A_{3}<0$. For $A_{3}=0$, (35) implies that $\omega=0$ is a double zero corresponding to Takens-Bogdanov bifurcation point. For oscillatory convection, we have

$$
\omega^{2}=\frac{-\left[\delta_{o}^{4}\left(M \Lambda+1 / \phi \operatorname{Pr}_{1}\right)+M \delta_{o}^{2} / D_{a}+Q \pi^{2}\left(M-\phi \operatorname{Pr}_{2} / \operatorname{Pr}_{1}\right)\right]}{\left[\delta_{o}^{2}\left(\operatorname{Pr}_{2} / \operatorname{Pr}_{1}\right)^{2}\left(\phi^{2} \Lambda / M+\phi / M^{2} \operatorname{Pr}_{1}\right)+\phi^{2} \operatorname{Pr}_{2}^{2} / M D_{a} \operatorname{Pr}_{1}^{2}\right]}
$$

where $\delta_{o}^{2}=\pi^{2}+q_{o}^{2}$. A necessary condition for $\omega^{2}>0$ is

$$
\frac{\operatorname{Pr}_{2}}{\operatorname{Pr}_{1}}>\frac{1}{\phi}
$$

However, this is not sufficient condition and one must have in addition

$$
Q>\frac{\delta_{o}^{4}\left(M \Lambda+1 / \phi \operatorname{Pr}_{1}\right)+M \delta_{o}^{2} / D_{a}}{\pi^{2}\left(\phi \operatorname{Pr}_{2} / \operatorname{Pr}_{1}-M\right)}
$$

At Takens-Bogdanov bifurcation point $R_{o}\left(q_{o}\right)=R_{s}\left(q_{s}\right)=$ $R_{c}\left(q_{c}\right), q_{o}=q_{s}=q_{c}$, and $\omega^{2}=0$ is a double zero at $Q=Q_{c}\left(q_{c}\right)$ where

$$
Q=\frac{\delta_{c}^{4}\left(M \Lambda+1 / \phi \operatorname{Pr}_{1}\right)+M \delta_{c}^{2} / D_{a}}{\pi^{2}\left(\phi \operatorname{Pr}_{2} / \operatorname{Pr}_{1}-M\right)}, \quad q=q_{c} .
$$

The Takens-Bogdanov bifurcation point occurs when the neutral curves for Hopf and pitchfork bifurcation meet and only a single wave number is present, namely, $q_{o}=q_{s}=q_{c}$. If $q_{c}>q_{\mathrm{sc}}$ then for all $q<q_{c}$ the first instability to set in is an oscillatory convection. The asymptotic values of $q_{c}$ and $q_{\mathrm{sc}}$ for large Chandrasekhar number $(Q \rightarrow \infty)$ are

$$
q_{c} \longrightarrow\left[\frac{\left(\phi \operatorname{Pr}_{2}-M \operatorname{Pr}_{1}\right) Q \pi^{2}}{\left(M \Lambda \operatorname{Pr}_{1}+1 / \phi\right)}\right]^{1 / 4}, \quad q_{\mathrm{sc}} \longrightarrow\left(\frac{Q \pi^{4} M}{2 \Lambda}\right)^{1 / 6} .
$$

From the monotonic dependence of $q_{c}$ and $q_{\mathrm{sc}}$ on $Q$, we may conclude that for $\operatorname{Pr}_{2}>\operatorname{Pr}_{1}$, there exists a $Q\left(\operatorname{Pr}_{1}, \operatorname{Pr}_{2}\right.$, $\left.M, \Lambda, \phi, D_{a}\right)$ such that for $Q<Q\left(\operatorname{Pr}_{1}, \operatorname{Pr}_{2}, M, \Lambda, \phi, D_{a}\right)$ the onset of first instability will be stationary convection at pitchfork bifurcation while for $Q>Q\left(\operatorname{Pr}_{1}, \operatorname{Pr}_{2}, M, \Lambda, \phi, D_{a}\right)$ it will be oscillatory convection at Hopf bifurcation. $Q\left(\operatorname{Pr}_{1}, \operatorname{Pr}_{2}\right.$, $\left.M, \Lambda, \phi, D_{a}\right)$ and for $Q=Q\left(\operatorname{Pr}_{1}, \operatorname{Pr}_{2}, M, \Lambda, \phi, D_{a}\right)$, we have

$$
R_{\mathrm{ct}}=R_{\mathrm{oc}}\left(q_{\mathrm{oc}}\right)=R_{\mathrm{sc}}\left(q_{\mathrm{sc}}\right) \quad \text { but } q_{\mathrm{oc}} \neq q_{\mathrm{sc}},
$$

above condition (41) gives codimension-two bifurcation point. However, there is no simple formula to give $Q\left(\operatorname{Pr}_{1}, \operatorname{Pr}_{2}\right.$, $\left.M, \Lambda, \phi, D_{a}\right)$ at the codimension-two bifurcation point by assuming $R$ as an independent variable, such kind of interesting result is not available in Chandrasekhar [2]. In Figures 1 and 2, each solid line stands for stationary convection (pitchfork bifurcation) and dotted line stands for oscillatory convection (Hopf bifurcation). In Figures 1 and 2, we have showed the effect of several physical parameters, like $Q$, $\operatorname{Pr}_{1}, \operatorname{Pr}_{2}, \Lambda, M, \phi$, and $D_{a}$ on the onset of both stationary convection and oscillatory convection when a physical parameter increases for the remaining fixed parameters, the onset of instabilities increases, that is, the onset of stationary convection and oscillatory convection inhibit when a parameter increases with the remaining fixed parameters.

\section{Onset of Stationary Convection at Supercritical Pitchfork Bifurcation}

4.1. Derivation of Two-Dimensional Nonlinear GinzburgLandau Equation Using Newell-Whitehead [19] Method. In this section the evolution of a general pattern is developed by means of a multiple scale analysis used by Newell and Whitehead [19]. A small amplitude convection cell is imposed on the basic flow. If this amplitude is of the size $O(\epsilon)$ then the interaction of the cell with itself forces a second harmonic and mean state correction of size $O\left(\epsilon^{2}\right)$ and then in turn drives an $O\left(\epsilon^{3}\right)$ correction to the fundamental component of the imposed roll. A solvability criteria for this correction yields the one-dimensional nonlinear Ginzburg-Landau equation of the complex valued amplitude $A(X, Y, T)$ of the imposed disturbance with real coefficients. To simplify the problem we assume the formulation of cylindrical rolls with axis parallel to $y$-axis, so that $y$-dependence disappears from (19). The $z$-dependence is contained entirely in the sine and cosine functions, which ensures that stress-free boundary conditions are satisfied. We use the expansion parameter $\epsilon$ as

$$
\epsilon^{2}=\frac{R-R_{\mathrm{sc}}}{R_{\mathrm{sc}}}
$$

For the values of $R$ close to threshold value $R_{\mathrm{sc}}$ that is, $\epsilon \ll 1$, the structure of the slow length scales will be insensitive to $\epsilon$, but a slow modulation in space and time is possible by making use of the band of the unstable solutions and linear growth rate is likely to saturate due to nonlinear effects. This behavior can be analyzed by writing solutions of (9)-(12) in power series $\epsilon$ as

$$
f=\epsilon f_{0}+\epsilon^{2} f_{1}+\epsilon^{3} f_{2}+\cdots,
$$

where $f=f\left(u, v, w, \theta, H_{x}, H_{y}, H_{z}\right)$ with the first approximation is given by the eigenvector of the linearized problem:

$$
\begin{gathered}
u_{0}=\frac{i \pi}{q_{\mathrm{sc}}}\left[A(X, Y, T) e^{i q_{\mathrm{sc}} x} \cos \pi z-\text { c.c. }\right], \\
v_{0}=0
\end{gathered}
$$




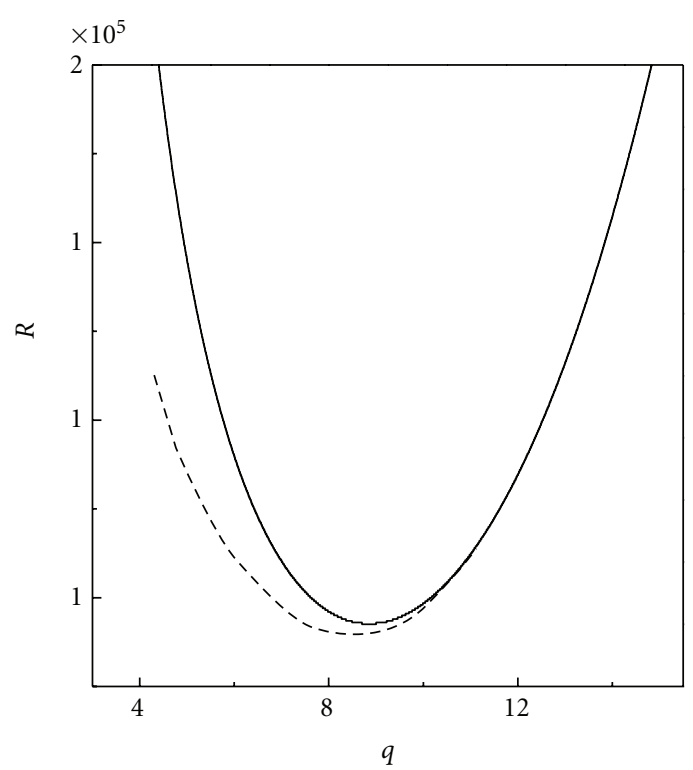

(a)

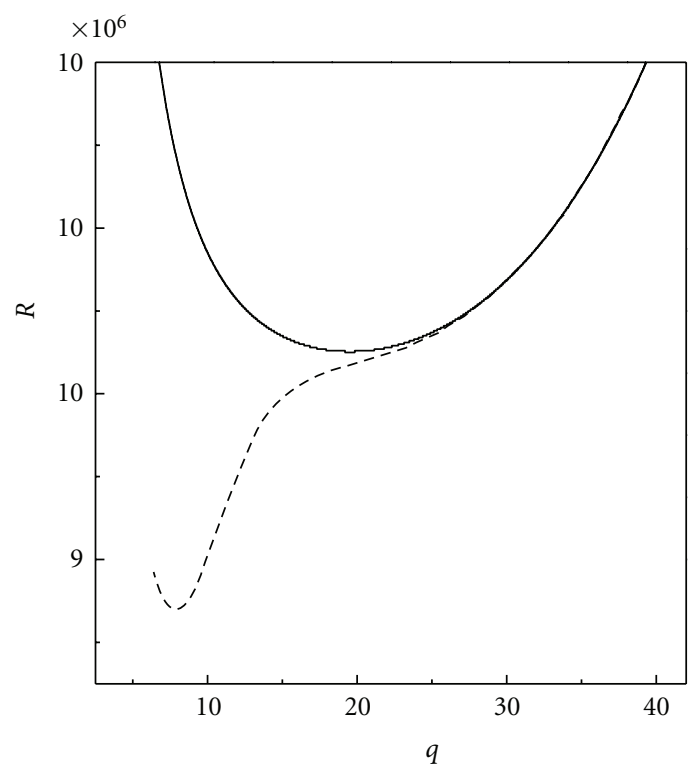

(c)

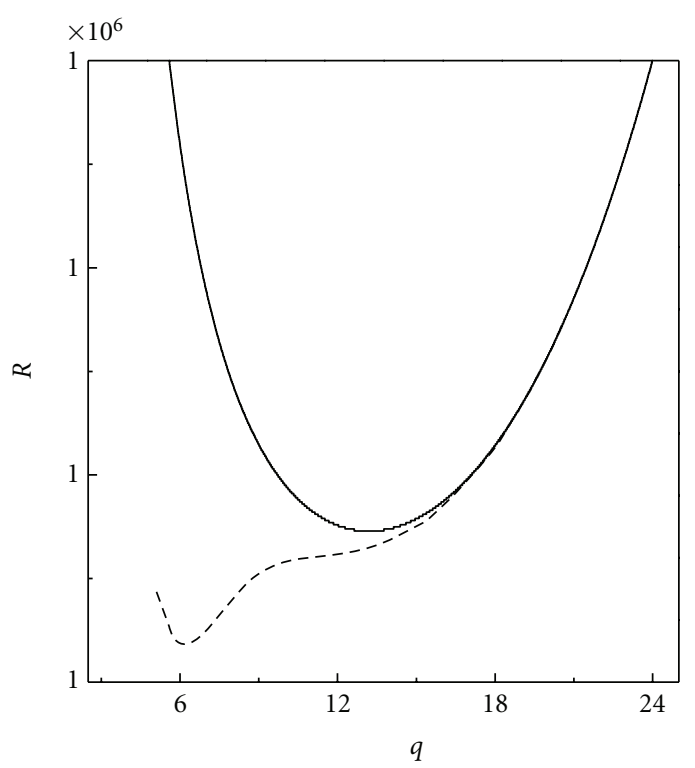

(b)

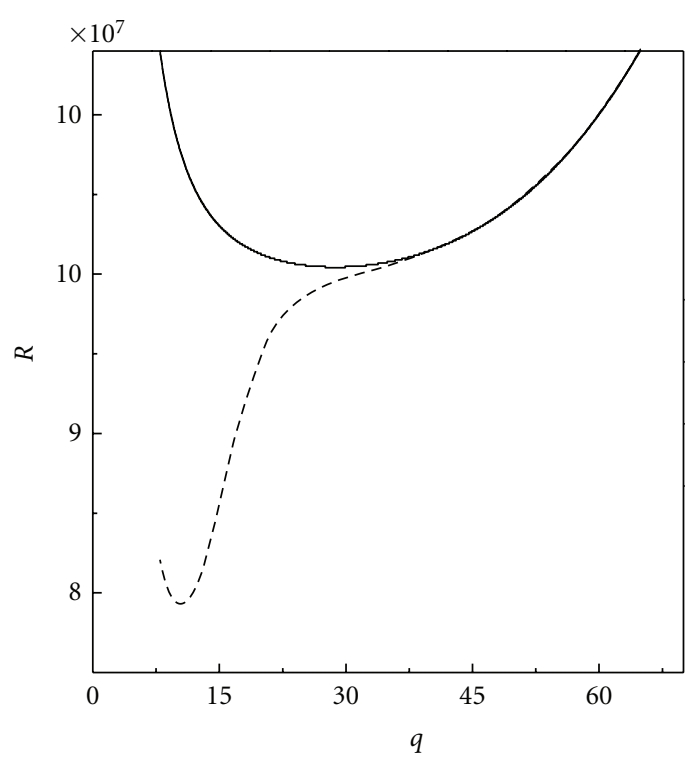

(d)

FIGURE 1: Numerically calculated marginal stability curves are plotted in $(R, q)$-plane for $\operatorname{Pr}_{1}=1, \operatorname{Pr}_{2}=1.5, D_{a}=1500, \Lambda=0.85, \phi=0.9$, and $M=0.9$, (a) $Q=10^{4}$, (b) $Q=10^{6}$, (c) $Q=10^{8}$, and (d) $Q=10^{10}$, then the onset of stationary convection and the onset of oscillatory convection increase (stationary convection represented by solid lines and oscillatory convection represented by dotted lines).

$$
\begin{gathered}
w_{0}=A(X, Y, T) e^{i q_{\mathrm{sc}} x} \sin \pi z+\text { c.c. }, \\
\theta_{0}=\frac{1}{M \delta_{\mathrm{sc}}^{2}}\left[A(X, Y, T) e^{i q_{\mathrm{sc}} x} \sin \pi z+\text { c.c. }\right], \\
H_{x_{0}}=\frac{-i \pi^{2}}{M q_{\mathrm{sc}} \delta_{\mathrm{sc}}^{2}}\left[A(X, Y, T) e^{i q_{\mathrm{sc}} x} \sin \pi z-\text { c.c. }\right], \\
H_{y_{0}}=0, \\
H_{z_{0}}=\frac{\pi}{M \delta_{\mathrm{sc}}^{2}}\left[A(X, Y, T) e^{i q_{\mathrm{sc}} x} \cos \pi z+\text { c.c. }\right],
\end{gathered}
$$

where $\delta_{\mathrm{sc}}^{2}=\pi^{2}+q_{\mathrm{sc}}^{2}$, here C.C. stands for complex conjugate, $e^{i q_{s c} x} \sin \pi z$ is the critical mode for the linear problem at $R=R_{\mathrm{sc}}$ and $q=q_{\mathrm{sc}}$. The complex amplitude $A(X, Y, T)$ depends on the slow variables $X, Y, Z$, and $T$ to be scaled by introducing multiple scales

$$
\begin{array}{cc}
X=\epsilon x, & Y=\epsilon^{1 / 2} y, \\
Z=z, & T=\epsilon^{2} t,
\end{array}
$$

and these formally separate the fast and slow dependent variables in $f$. It should be noted that the difference in scaling in the two directions reflects the inherent symmetry breaking 


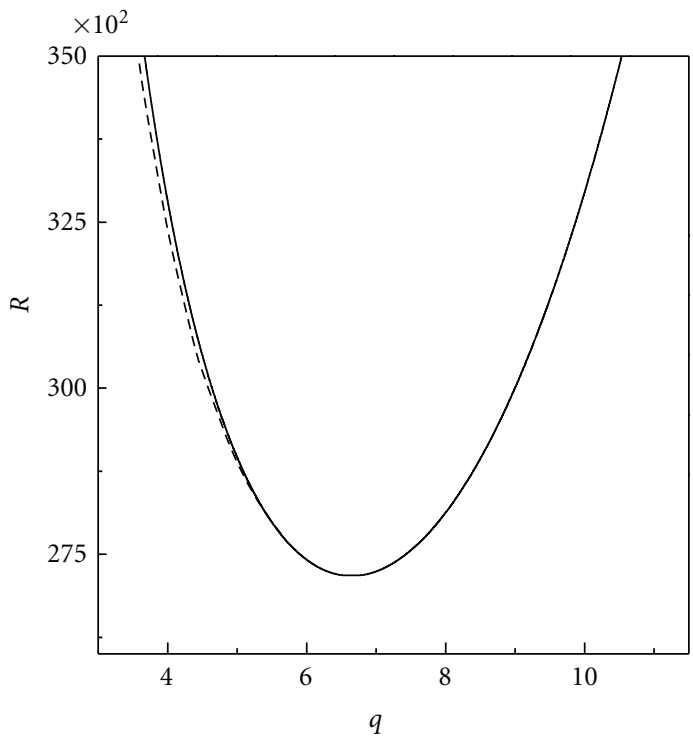

(a)

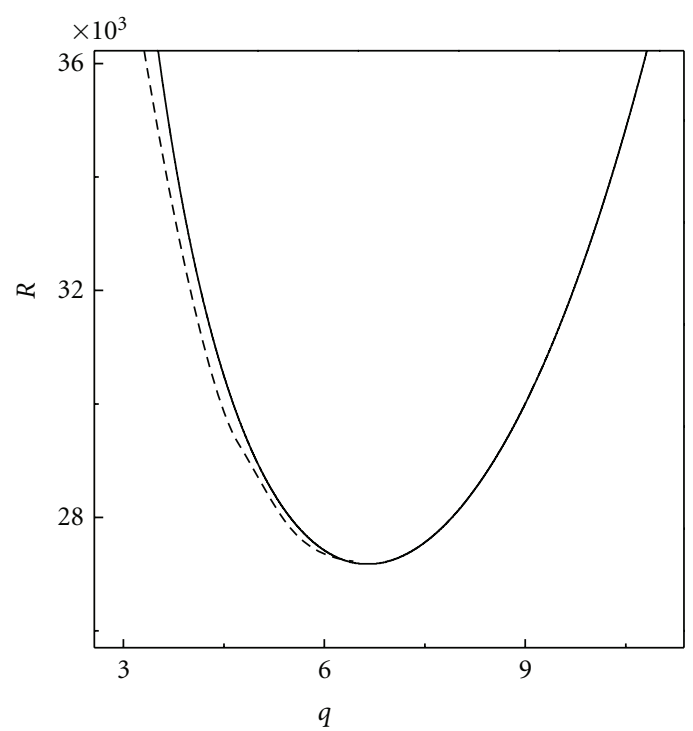

(b)

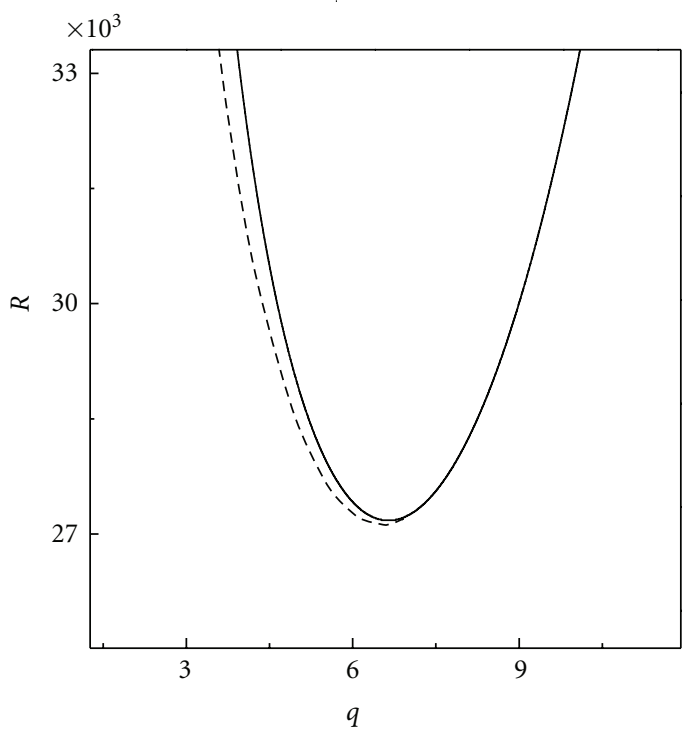

(c)

FIGURE 2: Neutral curves for the stationary bifurcation (solid lines) and for the Hopf bifurcation (dashed lines) near the codimension two point for $Q=2000, D_{a}=1500, \Lambda=0.85, \phi=0.9, \operatorname{Pr}_{1}=1$, and $M=0.9$, (a) $\operatorname{Pr}_{2}=1.27$, (b) $\operatorname{Pr}_{2}=1.3$, (c) $\operatorname{Pr}_{2}=1.35 x$-axis wave number, $y$-Rayleigh numbers $R_{s}, R_{o}$.

of instability which was chosen here with wave vector in $x$ direction. The differential operators can be expressed as

$$
\begin{gathered}
\frac{\partial}{\partial x} \longrightarrow \frac{\partial}{\partial x}+\epsilon \frac{\partial}{\partial X}, \quad \frac{\partial}{\partial y} \longrightarrow \epsilon^{1 / 2} \frac{\partial}{\partial Y}, \\
\frac{\partial}{\partial z} \longrightarrow \frac{\partial}{\partial Z}, \quad \frac{\partial}{\partial t} \longrightarrow \epsilon^{2} \frac{\partial}{\partial T}
\end{gathered}
$$

with the assumption (46), the operators (20) and (21) are transformed into a set of linear in homogeneous equations. The solvability conditions for the latter yields the amplitude equation using (44) in the linear operator (20) can be written as

$$
\mathcal{L}=\mathscr{L}_{0}+\epsilon \mathcal{L}_{1}+\epsilon^{2} \mathcal{L}_{2}+\cdots,
$$

where

$$
\begin{aligned}
\mathscr{L}_{0} & =-\Lambda \nabla^{8}+\frac{1}{D_{a}} \nabla^{6}+Q \nabla^{4} \frac{\partial^{2}}{\partial z^{2}}+R_{\mathrm{sc}} \nabla^{2} \frac{\partial^{2}}{\partial x^{2}}, \\
\mathscr{L}_{1}= & \left(2 \frac{\partial^{2}}{\partial x \partial X}+\frac{\partial^{2}}{\partial Y^{2}}\right) \\
& \times\left[\frac{3}{D_{a}} \nabla^{4}-4 \Lambda \nabla^{6}+2 Q \nabla^{2} \frac{\partial^{2}}{\partial z^{2}}+R_{\mathrm{sc}} \nabla^{2}+R_{\mathrm{sc}} \frac{\partial^{2}}{\partial x^{2}}\right],
\end{aligned}
$$




$$
\begin{aligned}
\mathcal{L}_{2}=\frac{\partial}{\partial T}[ & \left(\Lambda+\frac{1}{M \phi \operatorname{Pr}_{1}}+\phi \frac{\Lambda}{M} \frac{\operatorname{Pr}_{2}}{\operatorname{Pr}_{1}}\right) \nabla^{6} \\
& -\left(\frac{1}{D_{a}}+\phi \frac{\operatorname{Pr}_{2}}{\operatorname{Pr}_{1}} \frac{1}{M D_{a}}\right) \nabla^{4}-Q \nabla^{2} \frac{\partial^{2}}{\partial z^{2}} \\
& \left.-\frac{R_{\mathrm{sc}}}{M} \phi \frac{\operatorname{Pr}_{2}}{\operatorname{Pr}_{1}} \frac{\partial^{2}}{\partial x^{2}}\right]+\frac{\partial^{2}}{\partial X^{2}} \\
\times & {\left[\frac{3}{D_{a}} \nabla^{4}-4 \Lambda \nabla^{6}+2 Q \nabla^{2} \frac{\partial^{2}}{\partial z^{2}}+R_{\mathrm{sc}} \nabla^{2}+R_{\mathrm{sc}} \frac{\partial^{2}}{\partial x^{2}}\right] } \\
+ & \left(4 \frac{\partial^{4}}{\partial x^{2} \partial X^{2}}+\frac{1}{2} \frac{\partial^{2}}{\partial Y^{2}}\right) \\
\times & {\left[-6 \Lambda \nabla^{4}+\frac{3}{D_{a}} \nabla^{2}+Q \frac{\partial^{2}}{\partial z^{2}}+R_{\mathrm{sc}}\right] }
\end{aligned}
$$

Similarly nonlinear term $\mathcal{N}$ is given by

$$
\mathcal{N}=\epsilon^{2} \mathcal{N}_{0}+\epsilon^{3} \mathcal{N}_{1}+\cdots,
$$

substituting (47), (51), and (43) into (19), we get by equating the coefficients of $\epsilon, \epsilon^{2}, \epsilon^{3}$;

$$
\begin{gathered}
\mathcal{L}_{0} w_{0}=0, \\
\mathcal{L}_{0} w_{1}+\mathcal{L}_{1} w_{0}=\mathcal{N}_{0}, \\
\mathcal{L}_{0} w_{2}+\mathcal{L}_{1} w_{1}+\mathcal{L}_{2} w_{0}=\mathcal{N}_{1} .
\end{gathered}
$$

Equation (48) gives the critical Rayleigh number for the onset of stationary convection

$$
R_{\mathrm{sc}}=\frac{\delta_{\mathrm{sc}}^{2}}{q_{\mathrm{sc}}^{2}}\left[\delta_{\mathrm{sc}}^{4} \Lambda+\frac{1}{D_{a}} \delta_{\mathrm{sc}}^{2}+Q \pi^{2}\right] .
$$

In (53), $\mathcal{N}_{0}=0, \mathcal{L}_{1} w_{0}=0$ and hence $w_{1}=0$. From equation of continuity we find that $u_{1}=0$. The relevant equations for $\theta_{1}$ and $H_{z_{1}}$ are

$$
\left(\frac{\partial}{\partial t}-\nabla^{2}\right) \theta_{1}=\frac{w_{1}}{M}-\frac{1}{M}\left[u_{0} \frac{\partial \theta_{0}}{\partial x}+w_{0} \frac{\partial \theta_{0}}{\partial z}\right]
$$

form (56) and (44), we get

$$
\theta_{1}=-\frac{1}{2 \pi M^{2} \delta_{\mathrm{sc}}^{2}}|A|^{2} \sin 2 \pi z
$$

Equation (12) gives relevant equation for $H_{z_{1}}$ as

$$
\left(\phi \frac{\operatorname{Pr}_{2}}{\operatorname{Pr}_{1}}-M \nabla^{2}\right) H_{z_{1}}=\frac{\partial w_{1}}{\partial z}+\frac{\operatorname{Pr}_{2}}{\operatorname{Pr}_{1}} \frac{\partial}{\partial x}\left[w_{0} H_{x_{0}}-u_{0} H_{z_{0}}\right]
$$

From (58) and (44), we get

$$
H_{z_{1}}=\frac{\operatorname{Pr}_{2}}{\operatorname{Pr}_{1}} \cdot \frac{\pi^{2}}{4 \delta_{\mathrm{sc}}^{2} q_{\mathrm{sc}}^{2}}\left[A^{2} e^{2 i q_{\mathrm{sc}} x}+\text { c.c. }\right] \text {. }
$$

Similarly we have $H_{x_{1}}=0, H_{y_{1}}=0$. The solvability criterion of (54) gives the amplitude equation which can be written as

$$
\lambda_{0} \frac{\partial A}{\partial T}-\lambda_{1}\left(\frac{\partial}{\partial X}-\frac{i}{2 q_{\mathrm{sc}}} \frac{\partial^{2}}{\partial Y^{2}}\right)^{2}-\lambda_{2} A+\lambda_{3}|A|^{2} A=0
$$

where

$$
\begin{aligned}
\lambda_{0}= & \left(\Lambda+\frac{1}{M \phi \operatorname{Pr}_{1}}+\phi \frac{\operatorname{Pr}_{2}}{\operatorname{Pr}_{1}} \frac{\Lambda}{M}\right) \delta_{\mathrm{sc}}^{6}+\left(\frac{1}{D_{a}}+\phi \frac{\operatorname{Pr}_{2}}{\operatorname{Pr}_{1}} \frac{1}{M D_{a}}\right) \delta_{\mathrm{sc}}^{4} \\
& +Q \pi^{2} \delta_{\mathrm{sc}}^{2}-\frac{R_{\mathrm{sc}}}{M} \frac{\operatorname{Pr}_{2}}{\operatorname{Pr}_{1}} q_{\mathrm{sc}}^{2} \phi, \\
\lambda_{1}= & 4 q_{\mathrm{sc}}^{2}\left[6 \Lambda \delta_{\mathrm{sc}}^{4}+\frac{3}{D_{a}} \delta_{\mathrm{sc}}^{2}+Q \pi^{2}-R_{\mathrm{sc}}\right], \\
\lambda_{2}= & R_{\mathrm{sc}} q_{\mathrm{sc}}^{2} \delta_{\mathrm{sc}}^{2}, \\
\lambda_{3}= & Q \frac{\operatorname{Pr}_{2}^{2}}{\operatorname{Pr}_{1}^{2}} \frac{\pi^{4}}{M^{2} q_{\mathrm{sc}}^{2}}\left(q_{\mathrm{sc}}^{2}-\pi^{2}\right)+\frac{R_{\mathrm{sc}}}{2 M^{2}} q_{\mathrm{sc}}^{2} .
\end{aligned}
$$

Equation (60) is two-dimensional, nonlinear time-dependent Ginzburg-Landau equation describing the effect of magneticfield in a sparsely packed porous medium near the onset of stationary convection at supercritical pitchfork bifurcation. Here $\lambda_{0}$ is always positive for $\operatorname{Pr}_{2} / \operatorname{Pr}_{1}<1 / \phi$ and for any $Q$ but if $\operatorname{Pr}_{2} / \operatorname{Pr}_{1}>1 / \phi$ then $\lambda_{0}$ is positive only if $Q<Q_{c}$. Thus for supercritical pitchfork bifurcation $\lambda_{0}$ is always positive. For $\operatorname{Pr}_{2} / \operatorname{Pr}_{1}>1 / \phi, \lambda_{0}$ decreases as $Q$ increases and becomes zero at $Q=Q_{c}$. $\lambda_{1}$ and $\lambda_{2}$ are always positive. $\lambda_{3}$ is positive only if

$$
Q<\frac{R_{\mathrm{sc}} q_{\mathrm{sc}}^{4}}{2 \pi^{4}\left(\pi^{2}-q_{\mathrm{sc}}^{2}\right)} \cdot \frac{\operatorname{Pr}_{1}^{2}}{\operatorname{Pr}_{2}^{2}}
$$

The pitchfork bifurcation is supercritical if $\lambda_{3}>0$ and subcritical if $\lambda_{3}<0$. At $\lambda_{3}=0$, we get tricritical bifurcation point [22] (see Figure 3). Dropping the time dependence from (60), we get

$$
\frac{\mathrm{d}^{2} A}{\mathrm{~d} X^{2}}+\frac{\lambda_{2}}{\lambda_{1}}\left(1-\frac{\lambda_{3}}{\lambda_{2}}|A|^{2}\right) A=0,
$$

since $\lambda_{1}>0$, the solution of (63) is given by

$$
A(X)=A_{0} \tanh \left(\frac{X}{\Lambda_{1}}\right),
$$

where

$$
A_{0}=\left(\frac{\lambda_{2}}{\lambda_{3}}\right)^{1 / 2}, \quad \Lambda_{1}=\left(\frac{2 \lambda_{1}}{\lambda_{2}}\right)^{1 / 2}
$$

4.2. Long Wavelength Instabilities (Secondary Instabilities). The secondary Instabilities arising in nonequilibrium systems do not exhibit strict symmetries but may show spatially slow deformations of the cellular structures. Further, there are secondary instabilities like Eckhaus and Zigzag instabilities, such phenomena are studied using evolution 


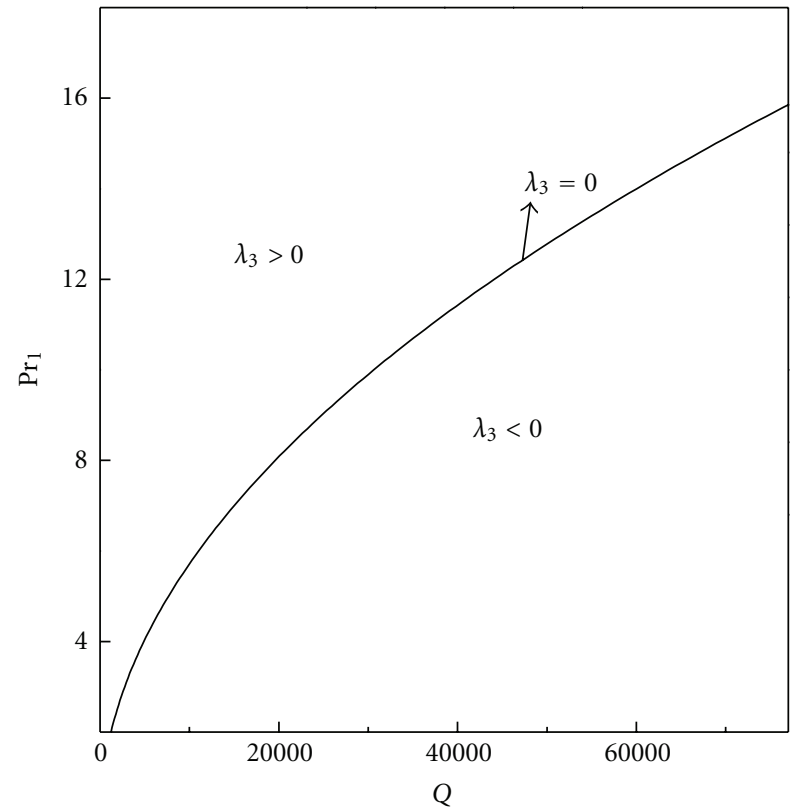

Figure 3: Above figure is plotted for $D_{a}=1500, \Lambda=0.85, \phi=$ $0.9, M=0.9$, and $\operatorname{Pr}_{2}=1 . \lambda_{3}$ is the nonlinear coefficient of Ginzburg-Landau equation at the onset of stationary convection. The pitchfork bifurcation is supercritical if $\lambda_{3}>0$, subcritical if $\lambda_{3}<0$ and $\lambda_{3}=0$ on the curve.

equations for amplitudes which are slowly varying in time as well as in space. These envelope equations can be derived by the method of Newell and Whitehead [19]. The twodimensional Ginzburg-Landau equation (60), can be written in fast variables $x, y, t$, and $A(X, Y, T)=A(x, y, t) / \epsilon$, as

$$
\lambda_{0} \frac{\partial A}{\partial t}-\lambda_{1}\left(\frac{\partial}{\partial x}-\frac{i}{2 q_{\mathrm{sc}}} \frac{\partial^{2}}{\partial y^{2}}\right)^{2} A-\epsilon^{2} \lambda_{2} A+\lambda_{3}|A|^{2} A=0
$$

In order to study the properties of a structure with a given phase winding number $\delta k$, we substitute

$$
A(x, y, t)=A_{1}(x, y, t) e^{i \delta k x}
$$

into (66) and we obtain

$$
\begin{aligned}
\lambda_{0} \frac{\partial A_{1}}{\partial t}= & \left(\epsilon^{2} \lambda_{2}-\lambda_{1}(\delta k)^{2}\right) A_{1} \\
& +2 i \lambda_{1} \delta k\left(\frac{\partial}{\partial x}-\frac{i}{2 q_{\mathrm{sc}}} \frac{\partial^{2}}{\partial y^{2}}\right) A_{1} \\
& +\lambda_{1}\left(\frac{\partial}{\partial x}-\frac{i}{2 q_{\mathrm{sc}}} \frac{\partial^{2}}{\partial y^{2}}\right)^{2} A_{1}-\lambda_{3}\left|A_{1}\right|^{2} A_{1}=0
\end{aligned}
$$

The steady-state uniform solution of $(68)$ is

$$
A_{1}=A_{1 o}=\left[\left(\epsilon^{2} \lambda_{2}-\lambda_{1}(\delta k)^{2}\right) \lambda_{3}^{-1}\right]^{1 / 2} .
$$

Let $\tilde{u}(x, y, t)+i \tilde{v}(x, y, t)$ be an infinitesimal perturbation from a uniform steady-state solution $A_{10}$ given by (69). Now substituting

$$
A_{1}=A_{1 o}=\left[\left(\epsilon^{2} \lambda_{2}-\lambda_{1}(\delta k)^{2}\right) \lambda_{3}^{-1}\right]^{1 / 2}+\tilde{u}+i \tilde{v},
$$

into (68) and equating real and imaginary parts, we obtain

$$
\begin{aligned}
\lambda_{0} \frac{\partial \tilde{u}}{\partial t}= & {\left[-2\left(\epsilon^{2} \lambda_{2}-\lambda_{1}(\delta k)\right)^{2}\right.} \\
& \left.+\lambda_{1}\left(\frac{\partial^{2}}{\partial x^{2}}+\frac{\delta k}{q_{\mathrm{sc}}} \frac{\partial^{2}}{\partial y^{2}}-\frac{1}{4 q_{\mathrm{sc}}^{2}} \frac{\partial^{4}}{\partial y^{4}}\right)\right] \tilde{u} \\
& -\left(2 \lambda_{1} \delta k-\frac{\lambda_{1}}{q_{\mathrm{sc}}} \frac{\partial^{2}}{\partial y^{2}}\right) \frac{\partial \tilde{v}}{\partial x}, \\
\lambda_{0} \frac{\partial \tilde{v}}{\partial t}= & \left(2 \lambda_{1} \delta k-\frac{\lambda_{1}}{q_{\mathrm{sc}}} \frac{\partial^{2}}{\partial y^{2}}\right) \frac{\partial \tilde{u}}{\partial x} \\
& +\lambda_{1}\left(\frac{\partial^{2}}{\partial x^{2}}+\frac{\delta k}{q_{\mathrm{sc}}} \frac{\partial^{2}}{\partial y^{2}}-\frac{1}{4 q_{\mathrm{sc}}^{2}} \frac{\partial^{4}}{\partial y^{4}}\right) \tilde{v} .
\end{aligned}
$$

We analyze (71) by using normal modes of the form

$$
\begin{aligned}
& \tilde{u}=U e^{S t} \cos \left(q_{x} x\right) \cos \left(q_{y} y\right), \\
& \tilde{v}=V e^{S t} \sin \left(q_{x} x\right) \cos \left(q_{y} y\right) .
\end{aligned}
$$

Putting (72) in (71) we get,

$$
\begin{gathered}
{\left[\lambda_{0} S+2\left(\epsilon^{2} \lambda_{2}-\lambda_{1}(\delta k)^{2}\right)+\chi_{1}\right] U+\lambda_{1} q_{x} \chi_{2} V=0,} \\
\lambda_{1} q_{x} \chi_{2} U+\left(\lambda_{0} S+\chi_{1}\right) V=0 .
\end{gathered}
$$

Here $\chi_{1}=\lambda_{1}\left[q_{x}^{2}+\left(q_{y}^{2} \delta k\right) / q_{\mathrm{sc}}+q_{y}^{4} / 4 q_{\mathrm{sc}}^{2}\right], \chi_{2}=\left(2 \delta k+q_{y}^{2} / q_{\mathrm{sc}}\right)$. On solving (73) we get,

$$
\begin{aligned}
\lambda_{0}^{2} S^{2} & +2 S\left[2 \lambda_{0}\left(\epsilon^{2} \lambda_{2}-\lambda_{1}(\delta k)^{2}\right)+\lambda_{0} \chi_{1}\right] \\
& +\left[2\left(\epsilon^{2} \lambda_{2}-\lambda_{1}(\delta k)^{2}\right)+\chi_{1}\right] \psi_{1}-q_{x}^{2} \lambda_{1} \chi_{2}=0,
\end{aligned}
$$

whose roots $(S \pm)$ are real. Here $(S \pm)$ is defined as

$$
\begin{aligned}
(S \pm)=-\frac{1}{\lambda_{0}^{2}}\{ & \left(2 \lambda_{0}\left(\epsilon^{2} \lambda_{2}-\lambda_{1}(\delta k)^{2}\right)+\lambda_{0} \chi_{1}\right) \\
& \left. \pm\left(2 \lambda_{0}\left(\epsilon^{2} \lambda_{2}-\lambda_{1}(\delta k)^{2}\right)^{2}+\lambda_{1}^{2} q_{x}^{2} \chi_{2}^{2}\right)^{1 / 2}\right\} .
\end{aligned}
$$

Solution $S(-)$ is clearly negative, thus the corresponding mode is stable and if $S(+)$ is positive then rolls can be unstable. Symmetry considerations help us to restrict the study of $S(+)$ to a domain $q_{x} \geq 0, q_{y} \geq 0$.

4.2.1. Longitudinal Perturbations and Eckhaus Instability. Inserting $q_{y}=0$ into (75), we get

$$
\begin{aligned}
\lambda_{0}^{2} S^{2} & +2 S\left[2 \lambda_{0}\left(\epsilon^{2} \lambda_{2}-\lambda_{1}(\delta k)^{2}\right)+\lambda_{0} \lambda_{1} q_{x}^{2}\right] \\
& +\lambda_{1} q_{x}^{2}\left[2\left(\epsilon^{2} \lambda_{2}-3 \lambda_{1}(\delta k)^{2}\right)+q_{x}^{2}\right]=0,
\end{aligned}
$$


since the roots are real and their sum always negative, the pattern is stable as long as both roots are negative, that is, their product is positive. The cell pattern becomes unstable when the product is negative, that is, when

$$
q_{x}^{2} \leq 2\left(3 \lambda_{1} \delta k^{2}-\epsilon^{2} \lambda_{2}\right),
$$

for this requires $|\delta k| \geq \sqrt{\left(\epsilon^{2} \lambda_{2} / 3 \lambda_{1}\right)}$, this condition defines the domain of Eckhaus instability. The above condition implies that the most unstable wave vector tends to zero, when $|\delta k| \rightarrow \sqrt{\left(\epsilon^{2} \lambda_{2} / 3 \lambda_{1}\right)}$.

4.2.2. Transverse Perturbations and Zigzag Instability. Let us consider $q_{x}=0$ into (75), we get

$$
\begin{aligned}
\lambda_{0}^{2} S^{2} & +2 S\left[2 \lambda_{0}\left(\epsilon^{2} \lambda_{2}-\lambda_{1}(\delta k)^{2}\right)+\lambda_{0} \chi_{1}^{y}\right] \\
& +\left[2\left(\epsilon^{2} \lambda_{2}-\lambda_{1}(\delta k)^{2}\right)+\chi_{1}^{y}\right] \chi_{1}^{y}=0,
\end{aligned}
$$

where $\chi_{1}^{y}=\lambda_{1}\left(q_{y}^{2} \delta k / q_{\mathrm{sc}}+q_{y}^{4} / 4 q_{\mathrm{sc}}^{2}\right)$. The two eigenmodes are uncoupled and we have $S(-)$,

$$
S(-)=-2\left(\epsilon^{2} \lambda_{2}-\lambda_{1}(\delta k)^{2}\right)-\frac{\lambda_{1}}{q_{\mathrm{sc}}} \delta k q_{y}^{2}-\frac{\lambda_{1}}{4 q_{\mathrm{sc}}^{2}} q_{y}^{2}<0,
$$

for one of them. The other is amplified when

$$
S(+)=-\lambda_{1} q_{y}^{2}\left(\delta k+\frac{q_{y}^{2}}{4 q_{\mathrm{sc}}}\right)>0 .
$$

This implies that $\delta k<0$, the above condition defines the domain of the Zigzag Instability. When $\delta k \rightarrow 0$ from below the wave vector $q_{y}$ of the instability also tends to zero while the growth rate varies as $q_{y}^{2}$. We have studied the effect of magnetic field on long wavelength instabilities. We have observed that Eckhaus instability and Zigzag instability regions increases when $Q$ increases (see Figure 4).

4.3. Heat Transport by Convection. The maximum of steady amplitude $A$ is denoted by $\left|A_{\max }\right|$ which is given as

$$
\left|A_{\max }\right|=\left(\frac{\epsilon^{2} \lambda_{2}}{\lambda_{3}}\right)^{1 / 2} .
$$

Equation (81) is obtained from (64) with $\tanh \left(X / \Lambda_{1}\right)=1$. We use $\left|A_{\max }\right|$ to calculate Nusselt number Nu. To discuss the heat transfer near the neutral region, we express it through the Nusselt number is defined as $\mathrm{Nu}=H d / \kappa \Delta T$, which is the ratio of the heat transported across any layer to the heat which would be transported by conduction alone. Here $H$ is the rate of heat transfer per unit area and is defined as

$$
H=-\left\langle\frac{\partial T_{\text {total }}}{\partial z^{\prime}}\right\rangle_{z^{\prime}=0} \text {. }
$$

In (82), angular brackets correspond to a horizontal average. The Nusselt number $\mathrm{Nu}$ can be calculated in terms of amplitude $A$ and is given as

$$
\mathrm{Nu}=1+\frac{\epsilon^{2}}{\delta_{\mathrm{sc}}^{2}}\left|A_{\max }\right|^{2} .
$$

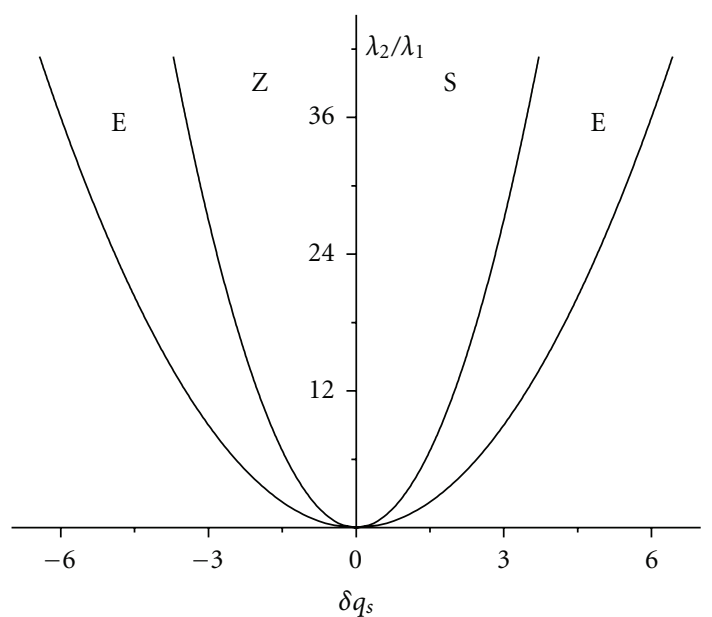

FIGURE 4: Numerically computed secondary instability regions of Eckhaus instability (E), Zigzag instability (Z), and stable regions (S) are plotted in $\left(\lambda_{2} / \lambda_{1}, \delta q_{s}\right)$-plane for $Q=2000, D_{a}=1500, \Lambda=0.85$, $\phi=0.9, M=0.9, \operatorname{Pr}_{1}=1$, and $\operatorname{Pr}_{2}=2$. As $\left|\delta q_{s}\right|$ increases then the secondary instability regions increases.

From (83), we get conduction for $R \leq R_{\mathrm{sc}}$ and convection for $R>R_{\mathrm{sc}}$. Since the amplitude equation is valid for $\lambda_{3}>$ 0 , which is possible for $R>R_{\mathrm{sc}}$ (supercritical pitchfork bifurcation), thus we get $\mathrm{Nu}>1$ for $R>R_{\mathrm{sc}}$. We get convection for $\mathrm{Nu}>1$ and conduction for $\mathrm{Nu} \leq 1$. In stationary convection $\mathrm{Nu}$ increases implies that heat conducted by steady mode increases. In the problem of double diffusive convection in porous medium with magnetic field, $\mathrm{Nu}$ depends on $\operatorname{Pr}_{1}, \operatorname{Pr}_{2}, \Lambda, M, \phi, D_{a}$, and $Q$. We have computed $\mathrm{Nu}$ for different values of $Q$, for some fixed values of other parameters and observed that $\mathrm{Nu}$ increases as $Q$ decreases (see Figures 5(a) and 5(b)). This implies that magnetic field inhibits the heat transport. The parameters $\operatorname{Pr}_{1}, \operatorname{Pr}_{2}, \Lambda, M, \phi$, and $D_{a}$ show the same result as $Q$ shows on $\mathrm{Nu}$.

\section{Oscillatory Convection at the Supercritical Hopf Bifurcation}

The existence of a threshold (critical value of Rayleigh number for the onset of oscillatory convection $R=R_{\mathrm{oc}}$ ) and a cellular structure (critical wave number $q=q_{\mathrm{oc}}$ ) are main characteristics of the oscillatory convection. In this section we treat the region near the onset of oscillatory convection. Here the axis of cylindrical rolls is taken as $y$-axis, so that $y$-dependence disappears from equation $\mathscr{L} w=\mathcal{N}$. The $z$ dependence contained entirely in sine and cosine functions which ensure that the free-free boundary conditions are satisfied. The purpose of this section is to derive coupled onedimensional nonlinear time-dependent Ginzburg-Landau type equations near the onset of oscillatory convection at supercritical Hopf bifurcation. We introduce $\epsilon$ as

$$
\epsilon^{2}=\frac{R_{o}-R_{\mathrm{oc}}}{R_{\mathrm{oc}}} \ll 1
$$




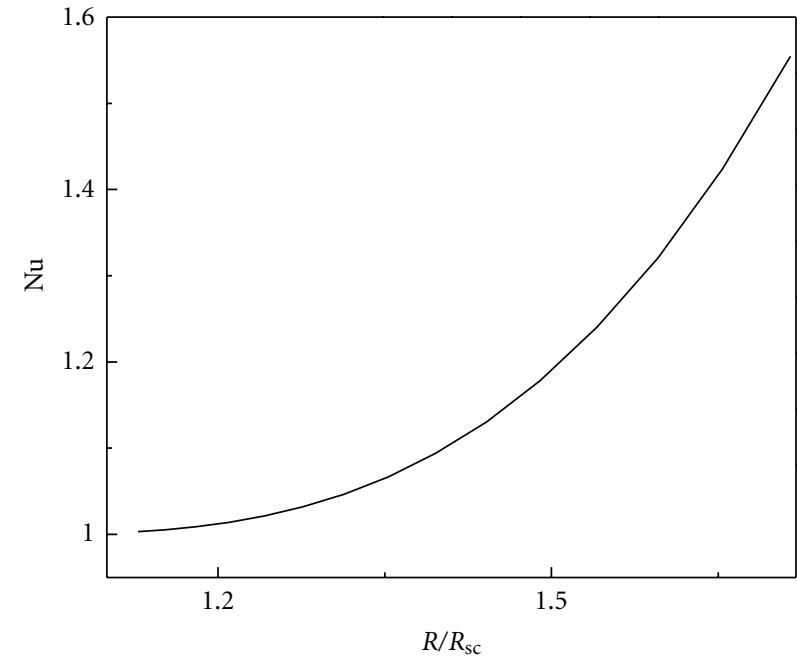

(a)

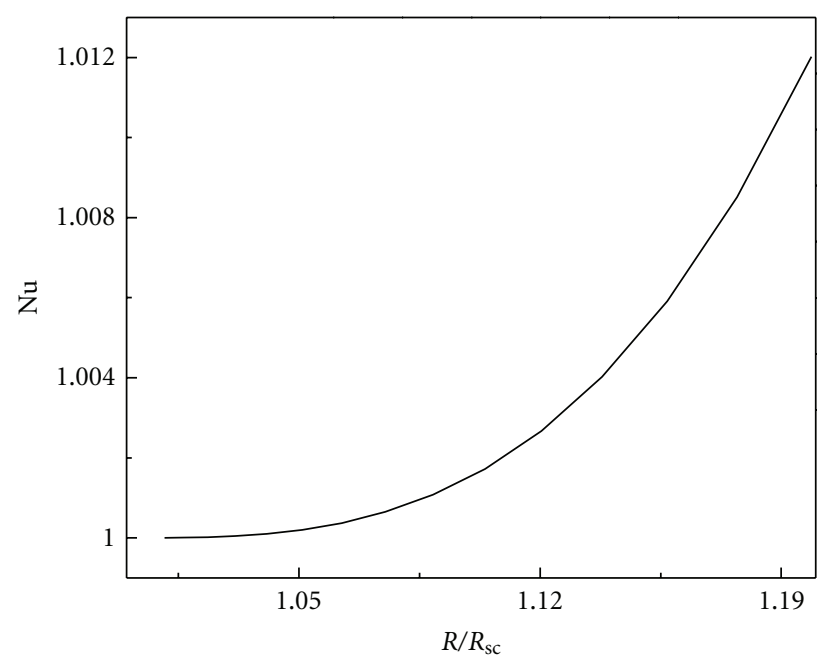

(b)

Figure 5: Graph (a) is plotted for $Q=1000$ and graph (b) is plotted for $Q=3000$ for the fixed values of $D_{a}=1500, \Lambda=0.85, \phi=0.9$, $\operatorname{Pr}_{2}=1, \operatorname{Pr}_{1}=2$, and $M=0.9$. in $\left(\mathrm{Nu}, R / R_{\mathrm{sc}}\right)$-plane. In graphs (a) and (b), as $R / R_{\mathrm{sc}}$ increases then $\mathrm{Nu}$ increases.

We assume that

$$
w_{0}=\left[A_{1 L} e^{i\left(q_{o c} x+\omega_{o c} t\right)}+A_{1 R} e^{i\left(q_{o c} x-\omega_{o c} t\right)}+\text { c.c. }\right] \sin \pi z
$$

is a solution to linearized equation $\mathcal{L} w=0$, which satisfies free-free boundary conditions. Here $A_{1 L}$ denotes the amplitude of left travelling wave of the roll and $A_{1 R}$ denotes the amplitude of right travelling wave of the roll, which depends on slow space and time variables [23]

$$
X=\epsilon x, \quad \tau=\epsilon t, \quad T=\epsilon^{2} t,
$$

and assume that $A_{1 L}=A_{1 L}(X, \tau, T), A_{1 R}=A_{1 R}(X, \tau, T)$. The differential operators can be expressed as

$$
\frac{\partial}{\partial x} \longrightarrow \frac{\partial}{\partial x}+\epsilon \frac{\partial}{\partial X}, \quad \frac{\partial}{\partial t} \longrightarrow \frac{\partial}{\partial t}+\epsilon \frac{\partial}{\partial \tau}+\epsilon^{2} \frac{\partial}{\partial T} .
$$

The solution of basic equations can be sought as power series in $\epsilon$,

$$
f=\epsilon f_{0}+\epsilon^{2} f_{1}+\epsilon^{3} f_{2}+\cdots,
$$

where $f=f\left(u, v, w, \theta, H_{x}, H_{y}, H_{z}\right)$ with the first approximation given by eigenvector of the linearized problem:

$$
\begin{aligned}
u_{0} & =\frac{i \pi}{q_{\mathrm{oc}}}\left[A_{1 L} e^{i\left(q_{\mathrm{oc}} x+\omega_{\mathrm{oc}} t\right)}+A_{1 R} e^{i\left(q_{\mathrm{oc}} x-\omega_{\mathrm{oc}} t\right)}-\text { c.c. }\right] \cos \pi z, \\
v_{0} & =0, \\
H_{y_{0}} & =0,
\end{aligned}
$$

$$
\begin{aligned}
\theta_{0}= & \frac{1}{M}\left[\frac{1}{e_{1}} A_{1 L} e^{i\left(q_{\mathrm{oc}} x+\omega_{\mathrm{oc}} t\right)}+\frac{1}{e_{1}^{*}} A_{1 R} e^{i\left(q_{\mathrm{oc}} x-\omega_{\mathrm{oc}} t\right)}+\text { c.c. }\right] \\
& \times \sin \pi z, \\
H_{x_{0}}= & \frac{-i \pi^{2}}{q_{\mathrm{oc}}}\left[\frac{1}{e_{2}} A_{1 L} e^{i\left(q_{\mathrm{oc}} x+\omega_{\mathrm{oc}} t\right)}+\frac{1}{e_{2}^{*}} A_{1 R} e^{i\left(q_{o c} x-\omega_{o c} t\right)}-\text { c.c. }\right] \\
& \times \sin \pi z, \\
H_{z_{0}=} & \pi\left[\frac{1}{e_{2}} A_{1 L} e^{i\left(q_{o c} x+\omega_{o c} t\right)}+\frac{1}{e_{2}^{*}} A_{1 R} e^{i\left(q_{o c} x-\omega_{o c} t\right)}+\text { c.c. }\right] \\
& \times \cos \pi z .
\end{aligned}
$$

where $\delta_{\mathrm{oc}}^{2}=\left(\pi^{2}+q_{\mathrm{oc}}^{2}\right), e_{1}=\left(\delta_{\mathrm{oc}}^{2}+i \omega_{\mathrm{oc}}\right)$, and $e_{2}=\left(M \delta_{\mathrm{oc}}^{2}+\right.$ $\left.i \omega_{\mathrm{oc}} \phi \operatorname{Pr}_{2} / \operatorname{Pr}_{1}\right)$, here $e_{1}^{*}$ and $e_{2}^{*}$ are complex conjugate of $e_{1}$ and $e_{2}$.

We expand the linear operator $\mathcal{L}$ and nonlinear term $\mathcal{N}$ as the following power series

$$
\begin{gathered}
\mathcal{L}=\mathscr{L}_{0}+\epsilon \mathcal{L}_{1}+\epsilon^{2} \mathcal{L}_{2}+\cdots, \\
\mathcal{N}=\epsilon^{2} \mathcal{N}_{0}+\epsilon^{3} \mathcal{N}_{1}+\cdots,
\end{gathered}
$$

substituting (87) and (88) into $\mathcal{L} w=\mathcal{N}$, for each order of $\epsilon$, we get

$$
\begin{gathered}
\mathcal{L}_{0} w_{0}=0, \\
\mathcal{L}_{0} w_{1}+\mathcal{L}_{1} w_{0}=\mathcal{N}_{0}, \\
\mathcal{L}_{0} w_{2}+\mathcal{L}_{1} w_{1}+\mathcal{L}_{2} w_{0}=\mathcal{N}_{1} .
\end{gathered}
$$


Here

$$
\begin{aligned}
& \mathcal{L}_{0}=\left(\mathscr{D}_{\phi} \mathscr{D}_{\mathrm{Pr}_{1}}-Q \frac{\partial^{2}}{\partial z^{2}}\right) \mathscr{D} \nabla^{2}-\frac{R}{M} \frac{\partial^{2}}{\partial x^{2}} \mathscr{D}_{\phi}, \\
& \mathcal{L}_{1}=\frac{\partial}{\partial \tau} \mathcal{F}_{1}+2 \frac{\partial^{2}}{\partial x X} \mathcal{F}_{2}, \\
& \mathcal{L}_{2}=\frac{\partial \mathcal{F}_{1}}{\partial T}+\frac{\partial^{4}}{\partial x^{2} X^{2}}\left[\left(M \nabla^{2}-\left(\mathscr{D}_{\phi}+M \mathscr{D}\right)\right) \mathscr{D}_{\operatorname{Pr}_{1}}\right. \\
& +\Lambda \mathscr{D} \nabla^{2}-\frac{\Lambda}{M} \mathscr{D} \mathscr{D}_{\phi}+\frac{\Lambda}{M} \mathscr{D}_{\phi} \nabla^{2} \\
& \left.+Q \frac{\partial^{2}}{\partial z^{2}}+R\right]+\frac{\partial^{2} \mathcal{F}_{2}}{\partial X^{2}}+2 \frac{\partial^{2}}{\partial x X} \frac{\partial}{\partial \tau} \\
& \times\left[\mathscr{D}_{\phi} \mathscr{D}_{\mathrm{Pr}_{1}}-\left(M+\phi \frac{\mathrm{Pr}_{2}}{\mathrm{Pr}_{1}}\right) \mathscr{D}_{\mathrm{Pr}_{1}} \nabla^{2}\right. \\
& -\left(\phi \frac{\operatorname{Pr}_{2}}{\operatorname{Pr}_{1}} \frac{\Lambda}{M}+\frac{1}{M \phi \operatorname{Pr}_{1}}\right) \mathscr{D} \nabla^{2}+\frac{1}{M^{2} \phi \operatorname{Pr}_{1}} D D_{\phi} \\
& +\phi \frac{\operatorname{Pr}_{2}}{\operatorname{Pr}_{1}} \mathscr{D} D_{\mathrm{Pr}_{1}}-\left(\frac{\Lambda}{M}+\frac{1}{M^{2} \phi \operatorname{Pr}_{1}}\right) \mathscr{D}_{\phi} \nabla^{2} \\
& \left.-Q \frac{\partial^{2}}{\partial z^{2}}-\frac{\phi R \operatorname{Pr}_{2}}{M \operatorname{Pr}_{1}}\right] \\
& +\frac{\partial^{2}}{\partial \tau^{2}}\left[\phi \frac{\operatorname{Pr}_{2}}{\operatorname{Pr}_{1}} \mathscr{D}_{\operatorname{Pr}_{1}} \nabla^{2}+\frac{\operatorname{Pr}_{2}}{M^{2} \operatorname{Pr}_{1}^{2}} \mathscr{D} \nabla^{2}\right. \\
& \left.+\frac{1}{M^{2} \phi \operatorname{Pr}_{1}} \mathscr{D}_{\phi} \nabla^{2}-\frac{R}{M} \nabla_{h}^{2} \mathscr{D}_{\phi}\right],
\end{aligned}
$$

where

$$
\begin{aligned}
& \mathscr{F}_{1}=\left(\mathscr{D}_{\phi} \mathscr{D}_{\mathrm{Pr}_{1}}+\phi \frac{\operatorname{Pr}_{2}}{\operatorname{Pr}_{1}} \mathfrak{D} \mathscr{D}_{\operatorname{Pr}_{1}}+\frac{1}{M^{2} \phi \operatorname{Pr}_{1}} \mathscr{D} \mathscr{D}_{\phi}\right) \nabla^{2} \\
& -Q \nabla^{2} \frac{\partial^{2}}{\partial z^{2}}-\frac{\phi R \operatorname{Pr}_{2}}{M \operatorname{Pr}_{1}} \nabla_{h}^{2}, \\
& \widetilde{F}_{2}=\left(\mathscr{D} \mathscr{D}_{\phi}-\mathscr{D}_{\phi} \nabla^{2}-M D^{2}\right) \mathcal{D}_{\mathrm{Pr}_{1}}-\frac{\Lambda}{M} \mathscr{D} D_{\phi} \nabla^{2} \\
& +Q \nabla^{2} \frac{\partial^{2}}{\partial z^{2}}-Q \mathcal{D} \frac{\partial^{2}}{\partial z^{2}}-\frac{R}{M} \mathcal{D}_{\phi}+R \nabla_{h}^{2} .
\end{aligned}
$$

Equation (91) is linear problem. We get critical Rayleigh number for the onset of oscillatory convection by using the zeroth-order solution $w_{0}$ in (91). At $O\left(\epsilon^{2}\right), \mathcal{N}_{0}=0$ and $\mathcal{L}_{1} w_{0}=0$ gives

$$
\frac{\partial A_{1 L}}{\partial \tau}-v_{g} \frac{\partial A_{1 L}}{\partial X}=0, \quad \frac{\partial A_{1 R}}{\partial \tau}-v_{g} \frac{\partial A_{1 R}}{\partial X}=0,
$$

where $v_{g}=(\partial \omega / \partial q)_{q=q_{o c}}$ is the group velocity and is real. Hence from (92), we get $w_{1}=0$. From equation of continuity we find that $u_{1}=0$. Substituting the zeroth-order and firstorder approximation into (56) and (58) we get,

$$
\begin{aligned}
& \theta_{1}= \frac{-\pi}{M^{2}}\left[\left(\left|A_{1 L}\right|^{2}+\left|A_{1 R}\right|^{2}\right) t_{1}+\frac{2}{e_{1} e_{4}} J_{1}+\frac{2}{e_{1}^{*} e_{4}^{*}} J_{1}^{*}\right] \sin 2 \pi z, \\
& v_{1}=0, \\
& H_{y_{1}}=0, \\
& H_{x_{1}}=\frac{i \pi}{2 M q_{\mathrm{oc}}} \frac{\operatorname{Pr}_{2}}{\operatorname{Pr}_{1}}\left[\left(\frac{1}{e_{2}}-\frac{1}{e_{2}^{*}}\right)\left(\left|A_{1 L}\right|^{2}-\left|A_{1 R}\right|^{2}\right)\right] \sin 2 \pi z, \\
& H_{z_{1}}=2 \pi^{2} \frac{\operatorname{Pr}_{2}}{\operatorname{Pr}_{1}}\left[\frac{1}{e_{2} e_{5}} A_{1 L}^{2} e^{2 i\left(q_{o c} x+\omega_{o c} t\right)}+\frac{1}{e_{2}^{*} e_{5}^{*}} A_{1 R}^{2} e^{2 i\left(q_{o c} x-\omega_{o c} t\right)}\right. \\
&\left.\quad+\frac{1}{4 M q_{\mathrm{oc}}^{2}}\left(\frac{1}{e_{2}}+\frac{1}{e_{2}^{*}}\right) A_{1 L} A_{1 R} e^{2 i q_{\circ \mathrm{oc}} x}+\text { c.c. }\right],
\end{aligned}
$$

where $t_{1}=\left(1 / 4 \pi^{2}\right)\left(1 / e_{1}+1 / e_{1}^{*}\right), J_{1}=A_{1 L} A_{1 R}^{*} e^{2 i \omega_{\mathrm{oc}} t}, e_{4}=$ $\left(4 \pi^{2}+2 i \omega_{\mathrm{oc}}\right)$, and $e_{5}=\left(4 M q_{\mathrm{oc}}^{2}+2 i \phi \omega_{\mathrm{oc}} \operatorname{Pr}_{2} / \operatorname{Pr}_{1}\right)$ and $e_{4}^{*}, e_{5}^{*}$ and $J_{1}^{*}$ are complex conjugate of $e_{4}, e_{5}$ and $J_{1}$, respectively.

Equation (93) is solvable when $\mathscr{L}_{0} w_{0}=0$, one requires that its right-hand side be orthogonal to $w_{0}$, which is ensured that if the coefficients of $\sin \pi z$ in $\mathcal{N}_{1}-\mathscr{L}_{2} w_{0}$ are equal to zero. This implies that

$$
\begin{gathered}
\Lambda_{0} \frac{\partial A_{1 L}}{\partial T}+\Lambda_{1}\left(\frac{\partial}{\partial \tau}-v_{g} \frac{\partial}{\partial X}\right) A_{2 L}-\Lambda_{2} \frac{\partial^{2} A_{1 L}}{\partial X^{2}}-\Lambda_{3} A_{1 L} \\
+\Lambda_{4}\left|A_{1 L}\right|^{2} A_{1 L}+\Lambda_{5}\left|A_{1 R}\right|^{2} A_{1 L}=0,
\end{gathered}
$$

$$
\begin{gathered}
\Lambda_{0} \frac{\partial A_{1 R}}{\partial T}+\Lambda_{1}\left(\frac{\partial}{\partial \tau}-v_{g} \frac{\partial}{\partial X}\right) A_{2 R}-\Lambda_{2} \frac{\partial^{2} A_{1 R}}{\partial X^{2}}-\Lambda_{3} A_{1 R} \\
+\Lambda_{4}\left|A_{1 R}\right|^{2} A_{1 R}+\Lambda_{5}\left|A_{1 L}\right|^{2} A_{1 R}=0,
\end{gathered}
$$

where

$$
\begin{aligned}
& \Lambda_{0}=\left(\frac{1}{M^{2} \phi \operatorname{Pr}_{1}} e_{1} e_{2}+e_{2} e_{3}+\phi \frac{\operatorname{Pr}_{2}}{\operatorname{Pr}_{1}} e_{1} e_{3}+Q \pi^{2}\right) \delta_{\mathrm{oc}}^{2} \\
&- \frac{R_{\mathrm{oc}} q_{\mathrm{oc}}^{2} \phi \operatorname{Pr}_{2}}{M \operatorname{Pr}_{1}}, \\
& \Lambda_{1}= \delta_{\mathrm{oc}}^{2}\left[e_{3} \phi \frac{\operatorname{Pr}_{2}}{\operatorname{Pr}_{1}}+\frac{e_{1} \operatorname{Pr}_{2}}{M^{2} \mathrm{Pr}_{1}^{2}}+\frac{e_{2}}{M^{2} \phi \operatorname{Pr}_{1}}\right], \\
& \Lambda_{2}=4 q_{\mathrm{oc}}^{2}\left[e_{2} e_{3}+M e_{3} \delta_{\mathrm{oc}}^{2}+\Lambda e_{1} \delta_{\mathrm{oc}}^{2}+\frac{\Lambda}{M} e_{1} e_{2}\right. \\
&\left.+M e_{1} e_{3}+\frac{\Lambda}{M} e_{2} \delta_{\mathrm{oc}}^{2}+Q \pi^{2}-R\right],
\end{aligned}
$$




$$
\begin{aligned}
\Lambda_{3}= & \frac{R}{M} q_{\mathrm{oc}}^{2} e_{2} \\
\Lambda_{4}= & -Q \frac{\pi^{4}}{M} \frac{\operatorname{Pr}_{2}^{2}}{\operatorname{Pr}_{1}^{2}} e_{1}\left(\frac{1}{e_{2}}-\frac{1}{e_{2}^{*}}\right) \\
& -2 Q \pi^{4} \frac{\operatorname{Pr}_{2}^{2}}{\operatorname{Pr}_{1}^{2}} \frac{e_{1}}{e_{5}}\left(\frac{1}{\delta_{\mathrm{oc}}^{2}}+\frac{\pi^{2}-3 q_{\mathrm{oc}}^{2}}{e_{2}^{*}}\right)+\frac{R}{M^{3}} \pi^{2} q_{\mathrm{oc}}^{2} t_{1} e_{2} \\
\Lambda_{5}= & Q \frac{\pi^{4}}{M} \frac{\operatorname{Pr}_{2}^{2}}{\operatorname{Pr}_{1}^{2}} e_{1}\left(\frac{1}{e_{2}}-\frac{1}{e_{2}^{*}}\right) \\
& -\frac{Q \pi^{4}}{M q_{\mathrm{oc}}^{2}} \frac{\operatorname{Pr}_{2}^{2}}{\operatorname{Pr}_{1}^{2}} e_{1}\left(\pi^{2}-q_{\mathrm{oc}}^{2}\right)\left(\frac{1}{e_{2}}+\frac{1}{e_{2}^{*}}\right) \\
& +\frac{R}{M^{3}} \pi^{2} q_{\mathrm{oc}}^{2} e_{2}\left(t_{1}+\frac{2}{e_{1} e_{4}}\right)
\end{aligned}
$$

Here $e_{3}=\left(i \omega / M^{2} \phi \operatorname{Pr}+(\Lambda / M) \delta^{2}+1 / M D_{a}\right)$. It should be noted that $A_{1 L}$ and $A_{1 R}$ are of order $\epsilon$ and $A_{2 L}$ and $A_{2 R}$ are of order $\epsilon^{2}$. If $\omega_{\text {oc }}=0$ in $\Lambda_{0}, \Lambda_{2}, \Lambda_{3}$, and $\Lambda_{4}$ then these expressions match with the coefficients $\lambda_{0}, \lambda_{1}, \lambda_{2}$, and $\lambda_{3}$ of GinzburgLandau equation at the onset of stationary convection. From (96), we get $A_{1 L}\left(\xi^{\prime}, T\right)$ and $A_{1 R}\left(\eta^{\prime}, T\right)$, where $\xi^{\prime}=v_{g} \tau+$ $X, \eta^{\prime}=v_{g} \tau-X$. Equations (98) can be written as

$$
\begin{aligned}
2 v_{g} \Lambda_{1} \frac{\partial A_{2 L}}{\partial \eta^{\prime}}= & -\Lambda_{0} \frac{\partial A_{1 L}}{\partial T}+\Lambda_{2} \frac{\partial A_{1 L}}{\partial X^{2}}+\lambda_{3} A_{1 L} \\
& -\left(\Lambda_{4}\left|A_{1 L}\right|^{2}+\Lambda_{5}\left|A_{1 R}\right|^{2}\right) A_{1 L}, \\
2 v_{g} \Lambda_{1} \frac{\partial A_{2 R}}{\partial \eta^{\prime}}= & -\Lambda_{0} \frac{\partial A_{1 R}}{\partial T}+\Lambda_{2} \frac{\partial A_{1 R}}{\partial X^{2}}+\lambda_{3} A_{1 R} \\
& -\left(\Lambda_{4}\left|A_{1 R}\right|^{2}+\Lambda_{5}\left|A_{1 L}\right|^{2}\right) A_{1 R} .
\end{aligned}
$$

Let $\xi^{\prime} \in\left[0, l_{1}\right], \eta^{\prime} \in\left[0, l_{2}\right]$ where $l_{1}, l_{2}$ are periods of $A_{1 L}$, $A_{1 R}$, respectively. Expansion (88) remains asymptotic for times $t=O\left(\epsilon^{-2}\right)$ only if an appropriate solvability condition holds. This condition obtained integrating (100) over $\eta^{\prime}$ and (101) over $\xi^{\prime}$, we get

$$
\begin{aligned}
\Lambda_{0} \frac{\partial A_{1 L}}{\partial T}= & \Lambda_{2} \frac{\partial A_{1 L}}{\partial X^{2}}+\lambda_{3} A_{1 L} \\
& -\left(\Lambda_{4}\left|A_{1 L}\right|^{2}+\Lambda_{5}\left|A_{1 R}\right|^{2}\right) A_{1 L} \\
\Lambda_{0} \frac{\partial A_{1 R}}{\partial T}= & \Lambda_{2} \frac{\partial A_{1 R}}{\partial X^{2}}+\lambda_{3} A_{1 R} \\
& -\left(\Lambda_{4}\left|A_{1 R}\right|^{2}+\Lambda_{5}\left|A_{1 L}\right|^{2}\right) A_{1 R}
\end{aligned}
$$

5.1. Travelling Wave and Standing Wave Convection. To study the stability regions of travelling waves and standing waves, Coullet et al. [24]. we proceed as follows.

On dropping slow variable $X$ from (102) and (103), we get a pair of first ODE's

$$
\frac{\mathrm{d} A_{1 L}}{\mathrm{~d} T}=\frac{\Lambda_{3}}{\Lambda_{0}} A_{1 L}-\frac{\Lambda_{4}}{\Lambda_{0}} A_{1 L}\left|A_{1 L}\right|^{2}-\frac{\Lambda_{5}}{\Lambda_{0}} A_{1 L}\left|A_{1 R}\right|^{2},
$$

$$
\frac{\mathrm{d} A_{1 R}}{\mathrm{~d} T}=\frac{\Lambda_{3}}{\Lambda_{0}} A_{1 R}-\frac{\Lambda_{4}}{\Lambda_{0}} A_{1 R}\left|A_{1 R}\right|^{2}-\frac{\Lambda_{5}}{\Lambda_{0}} A_{1 R}\left|A_{1 L}\right|^{2} .
$$

Put

$$
\beta^{\prime}=\frac{\Lambda_{3}}{\Lambda_{0}}, \quad \gamma^{\prime}=-\frac{\Lambda_{4}}{\Lambda_{0}}, \quad \delta^{\prime}=-\frac{\Lambda_{5}}{\Lambda_{0}} .
$$

Then (104) and (105) take the following form

$$
\begin{aligned}
& \frac{\mathrm{d} A_{1 L}}{\mathrm{~d} T}=\beta^{\prime} A_{1 L}+\gamma^{\prime} A_{1 L}\left|A_{1 L}\right|^{2}+\delta^{\prime} A_{1 L}\left|A_{1 R}\right|^{2}, \\
& \frac{\mathrm{d} A_{1 R}}{\mathrm{~d} T}=\beta^{\prime} A_{1 R}+\gamma^{\prime} A_{1 R}\left|A_{1 R}\right|^{2}+\delta^{\prime} A_{1 R}\left|A_{1 L}\right|^{2} .
\end{aligned}
$$

Consider $A_{1 L}=a_{L} e^{i \phi_{L}}$ and $A_{1 R}=a_{L} e^{i \phi_{R}}$ (we can write a complex number in the amplitude and phase form), where $a_{L}=\left|A_{1 L}\right|, \phi_{L}=\arg \left(A_{1 L}\right)=\tan ^{-1}\left(\operatorname{Im}\left(A_{1 L}\right) / \operatorname{Re}\left(A_{1 L}\right)\right)$ and $a_{R}=\left|A_{1 R}\right|, \phi_{R}=\arg \left(A_{1 R}\right)=\tan ^{-1}\left(\operatorname{Im}\left(A_{1 R}\right) / \operatorname{Re}\left(A_{1 R}\right)\right)$, here $a_{L}, a_{R}, \phi_{L}$, and $\phi_{R}$ are functions of time $T$ since $A_{1 L}$ and $A_{1 R}$ are functions of $T$. Thus $a_{L}$ and $a_{R}$ are positive functions. Substituting the definitions of $A_{1 L}$ and $A_{1 R}$ and $\beta^{\prime}=\beta_{1}+i \beta_{2}$, $\gamma^{\prime}=\gamma_{1}+i \gamma_{2}, \delta^{\prime}=\delta_{1}+i \delta_{2}$ into (107) and (108) we get,

$$
\begin{gathered}
\frac{\mathrm{d} a_{L}}{\mathrm{~d} T}=\beta_{1} a_{L}+\gamma_{1} a_{L}\left|a_{L}\right|^{2}+\delta_{1} a_{L}\left|a_{R}\right|^{2}, \\
\frac{\mathrm{d} \phi_{L}}{\mathrm{~d} T}=\beta_{2}+\gamma_{2}\left|a_{L}\right|^{2}+\delta_{2}\left|a_{R}\right|^{2}, \\
\frac{\mathrm{d} a_{R}}{\mathrm{~d} T}=\beta_{1} a_{R}+\gamma_{1} a_{R}\left|a_{R}\right|^{2}+\delta_{1} a_{R}\left|a_{L}\right|^{2}, \\
\frac{\mathrm{d} \phi_{R}}{\mathrm{~d} T}=\beta_{2}+\gamma_{2}\left|a_{R}\right|^{2}+\delta_{2}\left|a_{L}\right|^{2} .
\end{gathered}
$$

Equations (109) and (111) not contain phase term, so we take these two equations for the future discussions. We have (109) and (111) as

$$
\begin{aligned}
& \frac{\mathrm{d} a_{L}}{\mathrm{~d} T}=\beta_{1} a_{L}+\gamma_{1} a_{L}^{3}+\delta_{1} a_{R}^{2}, \\
& \frac{\mathrm{d} a_{R}}{\mathrm{~d} T}=\beta_{1} a_{R}+\gamma_{1} a_{R}^{3}+\delta_{1} a_{L}^{2},
\end{aligned}
$$

since $a_{L}$ and $a_{R}$ are positive functions. Put

$$
\frac{\mathrm{d} a_{L}}{\mathrm{~d} T}=F_{1}\left(a_{L}, a_{R}\right), \quad \frac{\mathrm{d} a_{R}}{\mathrm{~d} T}=F_{2}\left(a_{L}, a_{R}\right) .
$$

Now we discuss the stability of equilibrium points of (114). We get four equilibrium points like $\left(a_{L}, a_{R}\right)=(0,0)$ (conduction state), $\left(a_{L}, a_{R}\right)=\left(a_{L}, 0\right)\left(a_{L}=\right.$ amplitude of left travelling waves, here we get $F_{2}=0$, and we get one condition from $F_{1}=0$ i.e., $\left.a_{L}^{2}=-\beta_{1} / \gamma_{1}\left(=\left|A_{1 L}\right|^{2}\right)\right),\left(a_{L}, a_{R}\right)=(0$, $\left.a_{R}\right)\left(a_{R}=\right.$ amplitude of right travelling waves, here we get $F_{1}=0$, and we get one condition from $F_{2}=0$ i.e., $\left.a_{R}^{2}=-\beta_{1} / \gamma_{1}\left(=\left|A_{1 R}\right|^{2}\right)\right)$, and for $a_{L} \neq 0$ and $a_{R} \neq 0$ we get $\left(a_{L}, a_{R}\right)=\left(-\beta_{1} /\left(\gamma_{1}+\delta_{1}\right),-\beta_{1} /\left(\gamma_{1}+\delta_{1}\right)\right)$ (this gives condition for standing waves. At standing waves we have $A_{L}=A_{R}$, so $\left.a_{L}=a_{R}\right)$. For the pair of (104) and (105), we do not get 
$a_{L} \neq a_{R} \neq 0$ (modulated waves). Now the Jacobian of $F_{1}$ and $F_{2}$ is given by

$$
\left(\begin{array}{ll}
\frac{\partial F_{1}}{\partial a_{L}} & \frac{\partial F_{1}}{\partial a_{R}} \\
\frac{\partial F_{2}}{\partial a_{L}} & \frac{\partial F_{2}}{\partial a_{R}}
\end{array}\right) .
$$

If real parts of all eigenvalues of the Jacobian are negative at an equilibrium point, then that point is a stable equilibrium (Lyapunov's theorem or principle of linearized stability). Some valuable conditions for travelling waves and standing waves are travelling waves are stable if $\beta_{1}>0, \gamma_{1}<0$ and $\delta_{1}<\gamma_{1}<0$. Standing waves are stable if $\beta_{1}>0, \gamma_{1}<0$ and (i) if $\delta_{1}>0$, then $-\gamma_{1}>\delta_{1}>0$ and (ii) if $\delta_{1}<0$, then $-\gamma_{1}>-\delta_{1}>0$.

The stability regions of travelling waves and standing waves are summarized in Figure 6. Here $E$ is total amplitude and defined as $E=a_{L}^{2}+a_{R}^{2}$. We do not distinguish between left travelling waves and right travelling waves. For rest state (steady state) $E=0$, for travelling waves $E=-\beta_{1} / \gamma_{1}$, for standing waves $E=-2 \beta_{1} /\left(\gamma_{1}+\varsigma_{1}\right)$. Travelling waves are supercritical if $\gamma_{1}<0$ and standing waves are supercritical if $\gamma_{1}+\varsigma_{1}<0$. Figure $6(\mathrm{a})$ is drawn for stable travelling wave conditions and Figure 6(b) is drawn for stable standing wave conditions in $\left(\beta_{1}, E\right)$-plane. The symbols $(-,-)$ and $(+,+)$ in Figures 6(a) and 6(b) indicate that both roots of Jacobian are negative and at least one root is positive between two roots. In Figures 6(a) and 6(b), travelling wave solution and standing wave solution bifurcate simultaneously from the steady-state solution ( $\beta_{1} \geq 0$ at this bifurcation point).

In these Figures $6(\mathrm{a})$ and $6(\mathrm{~b})$, steady-state solution is stable for $\beta_{1}<0$ and unstable $\beta_{1}>0$. These figures show that for $\beta_{1}>0$ both travelling waves and standing waves are supercritical. When travelling waves and standing waves bifurcate supercritically then at most one solution among travelling waves and standing waves will be stable. Thus, for $\beta_{1}>0$ (Figure 6(a)) travelling waves are stable and (Figure 6(b)) standing waves are stable. In more detail we reproduce results of the stability analysis of equilibrium solutions in Figure 6(c), which is plotted in $\left(\gamma_{1}, \varsigma_{1}\right)$-plane. From this figure we can observe that travelling waves are subcritical for $\gamma_{1}>0$ and standing waves are subcritical for $\gamma_{1}+\varsigma_{1}>0$. In Figure 7, We study the stability regions of travelling waves and standing waves at the onset of Hopf bifurcation. The stability regions of standing waves and travelling waves increases when $\mathrm{Pr}_{2} / \mathrm{Pr}_{1}$ increases for fixed parameters. For a fixed $\operatorname{Pr}_{1}$ if we get initially travelling waves at the onset of oscillatory convection then they are replaced by standing waves as $Q$ increases.

5.2. Long Wavelength Instabilities for the Onset of Travelling Wave Convection (Benjamin-Feir Instability). For right travelling wave $A_{R}(X, T)=A(X, T)$ and $A_{L}(X, T)=0$, for left travelling wave $A_{R}(X, T)=0$ and $A_{L}(X, T)=A(X, T)$. Thus for travelling waves we get a single amplitude equation from (102) and (103), given as

$$
\Lambda_{0} \frac{\partial A}{\partial T}-\Lambda_{2} \frac{\partial^{2} A}{\partial X^{2}}-\Lambda_{3} A+\Lambda_{4}|A|^{2} A=0 .
$$

For standing waves $A_{1 L}(X, T)=A_{1 R}(X, T)=A(X, T)$ and we get a single amplitude equation from (102) and (103), given as

$$
\Lambda_{0} \frac{\partial A}{\partial T}-\Lambda_{2} \frac{\partial^{2}}{\partial X^{2}} A-\Lambda_{3} A+\left(\Lambda_{4}+\Lambda_{5}\right)|A|^{2} A=0 .
$$

Equation (117) possesses a family of planar wave solutions and solutions containing phase singular points, which describes weakly nonlinear wave phenomena [25]. We study the Benjamin-Feir instability of travelling waves from complex Ginzburg- Landau equation (116) can be written as

$$
\frac{\partial A}{\partial T}=\xi \frac{\partial^{2} A}{\partial X^{2}}+\beta A+\gamma|A|^{2} A,
$$

where $\xi=\xi_{1}+i \xi_{2}, \beta=\beta_{1}+i \beta_{2}, \gamma=\gamma_{1}+i \gamma_{2}$. The phase winding solutions are obtained by substituting $A=$ $\widetilde{A}_{o} e^{i\left(\delta q_{o} X-\delta \omega T\right)}$ into (118), and equating real and imaginary parts we get

$$
\begin{gathered}
\left|\tilde{A}_{o}\right|^{2}=\xi_{1} \delta q_{o}^{2}-\beta_{1} \gamma_{1}^{-1} \\
\delta \omega=\xi_{2} \delta q_{o}^{2}-\beta_{2}+\gamma_{2}\left(\beta_{1}-\xi_{1} \delta q_{o}^{2}\right) \gamma_{1}^{-1} .
\end{gathered}
$$

Here $\tilde{A}_{o}$ is constant and $\delta q_{o}=q X-q_{\mathrm{oc}}$. We consider a modulated solution in the form: $A(X, T)=\widetilde{A}(X, T) e^{i\left(\delta q_{0} X-\delta \omega T\right)}$. Substituting the modulated into (118) which gives

$$
\begin{aligned}
\frac{\partial \tilde{A}}{\partial T}= & \left(\gamma_{1}+i \gamma_{2}\right)\left[\frac{\left(\beta_{1}-\delta q_{o}^{2} \xi_{1}\right)}{\gamma_{1}}+|\tilde{A}|^{2}\right] \tilde{A} \\
& +\left(\gamma_{1}+i \gamma_{2}\right)\left(\frac{\partial^{2}}{\partial X^{2}}+2 i \delta q_{o} \frac{\partial}{\partial X}\right) \tilde{A} .
\end{aligned}
$$

It is possible to conduct a general investigation of the linear stability of $A(X, T)$, but this is very difficult task, and therefore our primary concern here is to treat the stability of the uniformly oscillating solution $\widetilde{A}_{o}$. Inserting $\widetilde{A}=\widetilde{A}_{o}+\tilde{u}+$ $i \tilde{v}$ into (120) and equating real and imaginary parts we get

$$
\begin{aligned}
\frac{\partial \tilde{u}}{\partial T}= & -2\left(\beta_{1}-\delta q_{o}^{2} \xi_{1}\right) \tilde{u}+\xi_{1}\left(\frac{\partial^{2} \tilde{u}}{\partial X^{2}}-2 \delta q_{o} \frac{\partial \tilde{v}}{\partial X}\right) \\
& -\xi_{2}\left(2 \delta q_{o} \frac{\partial \tilde{u}}{\partial X}+\frac{\partial \tilde{v}}{\partial X^{2}}\right), \\
\frac{\partial \tilde{v}}{\partial T}= & \frac{-2 \gamma_{2}\left(\beta_{1}-\delta q_{o}^{2} \xi_{1}\right)}{\gamma_{1}} \tilde{u}+\xi_{1}\left(2 \delta q_{o} \frac{\partial \tilde{u}}{\partial X}+\frac{\partial^{2} \tilde{v}}{\partial X^{2}}\right) \\
& +\xi_{2}\left(\frac{\partial^{2} \tilde{u}}{\partial X^{2}}-2 \delta q_{o} \frac{\partial \tilde{v}}{\partial X}\right) .
\end{aligned}
$$

Consider $(\tilde{u}, \tilde{v})=(U, V) e^{S T} \cos q_{X} X$ and $S$ in the growth rate of disturbances. Using solutions of $\tilde{u}, \tilde{v}$, and $\delta q_{0}=0$ into (121) and (122) we get,

$$
\begin{gathered}
\left(S+2 \beta_{1}+\xi_{1} q_{X}^{2}\right) U-q_{X}^{2} \xi_{2} V=0, \\
\left(S+q_{X}^{2} \xi_{1}\right) V+\left(2 \beta_{1} \gamma_{2} \gamma_{1}^{-1}+q_{X}^{2} \xi_{2}\right) U=0
\end{gathered}
$$




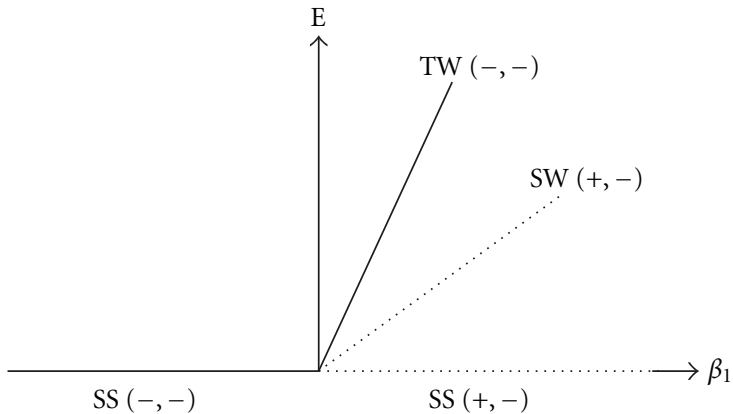

(a)

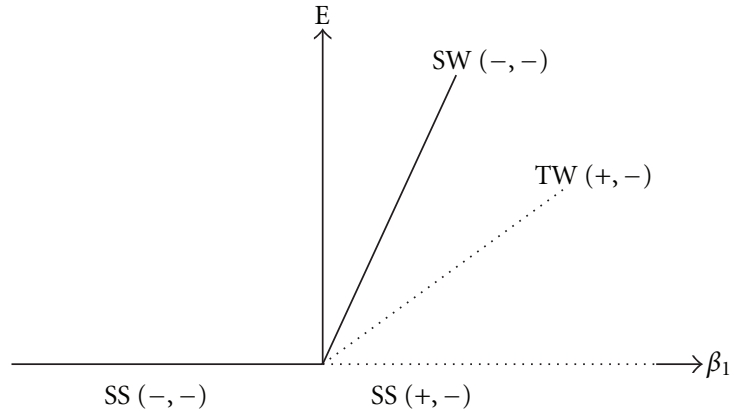

(b)

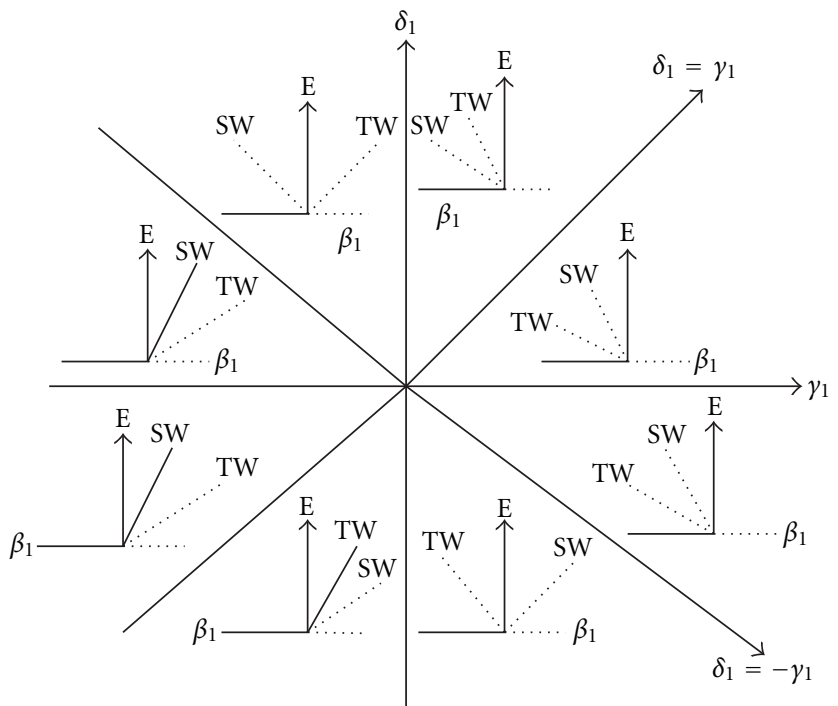

(c)

FIgURE 6: (a, b, c) are typical diagrams showing the stability of equilibrium solutions SS (steady state), SW (standing waves), and TW (travelling waves). On solid lines equilibrium solutions are stable and on dotted lines they are unstable.

solving (123) and (124), we get

$$
\begin{aligned}
S^{2}+ & 2 S\left(\beta_{1}+\xi_{1} q_{X}^{2}\right)+q_{X}^{2} \xi_{1}\left(2 \beta_{1}+\xi_{1} q_{X}^{2}\right) \\
& +q_{X}^{2} \xi_{2}\left(2 \beta_{1} \gamma_{2} \gamma_{1}^{-1}+q_{X}^{2} \xi_{2}\right) .
\end{aligned}
$$

There will be an instability only when a root of (125) is possible, that is,

$$
2 \beta_{1}\left(\xi_{1}+\gamma_{2} \xi_{2} \gamma_{1}^{-1}\right)+q_{X}^{2}\left(\xi_{1}^{2}+\xi_{2}^{2}\right)<0,
$$

$\beta_{1}>0$ when travelling waves or standing waves are stable. The instability of waves against long wavelength longitudinal modes is often called the Benjamin-Feir instability. Thus we get Benjamin-Feir instability for travelling waves when $\xi_{1}+\gamma_{2} \xi_{2} / \gamma_{1}<0$. Similarly by considering (118) instead of (117) and proceeding in the same way we get Benjamin-Feir instability for standing waves when $\xi_{1}+\left(\gamma_{2}+\delta_{2}\right) \xi_{2} /\left(\gamma_{1}+\delta_{1}\right)<$ 0 .

\section{Conclusions}

In this paper we have considered both linear and weakly nonlinear analysis of magnetoconvection in a sparsely packed porous medium in Earth's outer core by using free-free (stress-free) boundary conditions. Even though free-free boundary conditions cannot be achieved in laboratory, one can use it in geophysical fluid dynamic applications to Earth's outer core since they allow simple trigonometric eigenfunctions. Our goal is to identify the region of parameter values, for which rolls emerge at the onset of convection.

Following Chandrasekhar [2], we have described the stationary convection and oscillatory convection as curves $R_{s}(q)$ and $R_{o}\left(q, \operatorname{Pr}_{2}\right)$ versus wave numbers. The critical wave numbers for stationary convection and oscillatory convection are $q_{\mathrm{sc}}=q_{\mathrm{oc}}=\pi / \sqrt{2}$. For the problem of magnetoconvection in a sparsely packed porous medium, we get Takens-Bogdanov bifurcation point and codimensiontwo bifurcation point. In the case of linear theory both marginal and overstable motions are discussed. In Figures 1 and 2, is shown that the effect of Chandrasekhar number and porous parameter is to make the system more stable. By drawing stability boundaries in the Rayleigh number plane it is shown that the effect of magnetic field and porous parameter is to decrease the region of stabilities. 


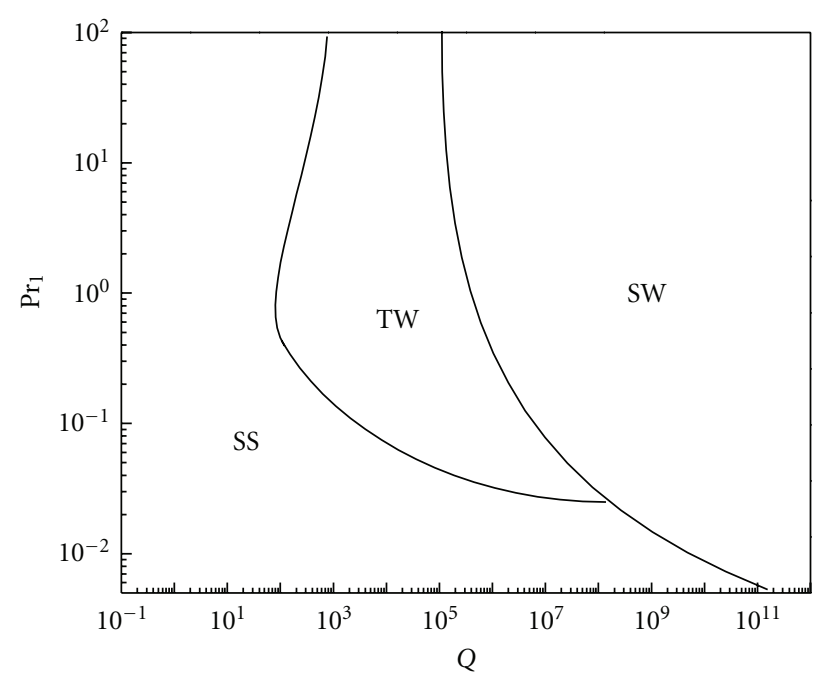

(a)

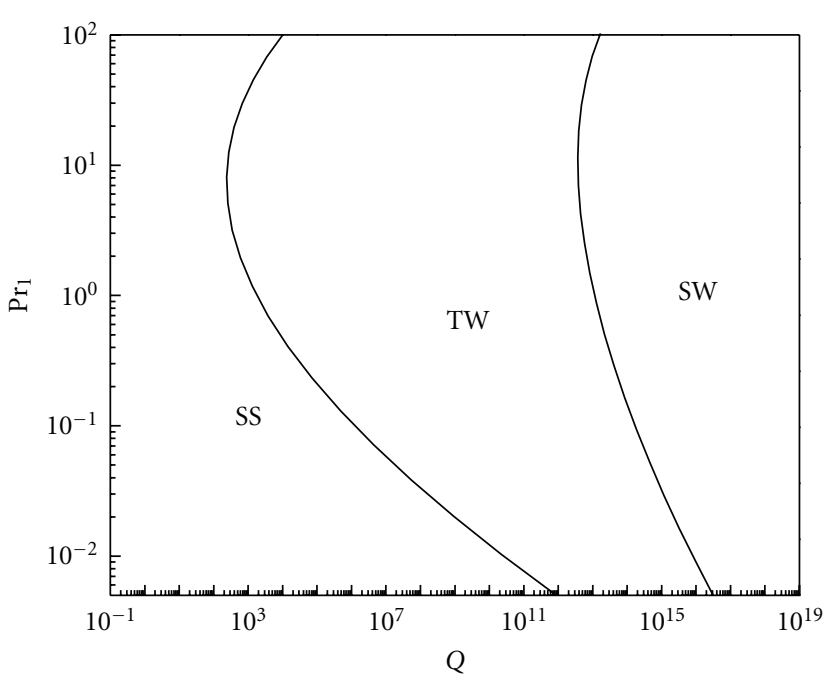

(c)

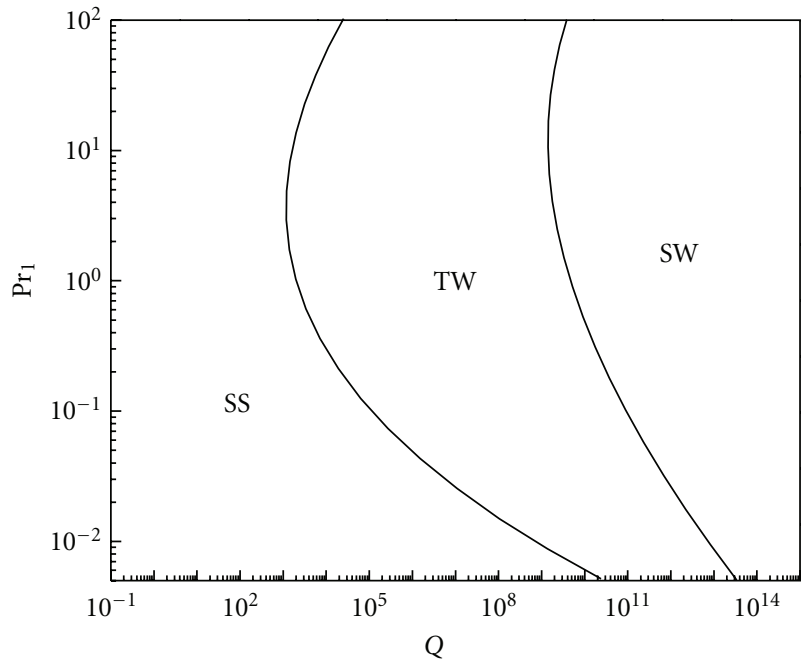

(b)

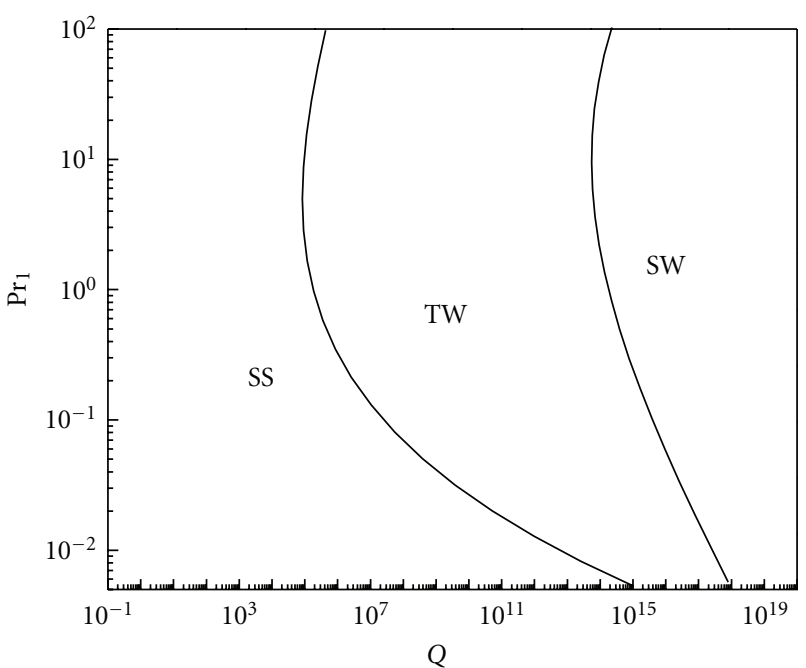

(d)

Figure 7: Figures (a-d), are plotted for $\operatorname{Pr}_{2} / \operatorname{Pr}_{1}=6,12,18$, and 24, respectively, and for fixed parameters $D_{a}=1500, \Lambda=0.85, \phi=0.9$, and $M=0.9$. Stability regions of steady state (SS), travelling waves (TW), and standing waves (SW) are plotted $\left(Q, \operatorname{Pr}_{1}\right)$-plane.

In the nonlinear equation (60), $\lambda_{0}=0$ gives the TakensBogdanov bifurcation point at $q_{s}=q_{\mathrm{sc}}$ and when $\lambda_{0}=0$, (60) is not valid. The pitchfork bifurcation is supercritical if $\lambda_{3}>0$ subcritical if $\lambda_{3}<0$. and we get tricritical point if $\lambda_{3}=0$. We have obtained from (60), long wave length instabilities, namely, Eckhaus and Zigzag instabilities. From (60) which is valid only for $\lambda_{3}>0$, we have calculated Nusselt number $\mathrm{Nu}$ and studied heat transport by convection. We have also derived two one-dimensional nonlinear coupled Ginzburg-Landau type equations, namely, (98) at the onset of oscillatory convection at supercritical Hopf bifurcation. We have computed stability regions of SW and TW at both Hopf bifurcation. The conditions for SW and TW are $A_{L}=$ $A_{R}$ and $A_{L}=0$ or $A_{R}=0$, respectively. TW exist if $\left|A_{L}\right|^{2}=$ $-\beta_{1} / \gamma_{1}>0$ and they are supercritical if $\gamma_{1}<0$. SW exist if
$\left|A_{L}\right|^{2}=\left|A_{R}\right|^{2}=-\beta_{1} /\left(\gamma_{1}+\delta_{1}\right)>0$ and SW are supercritical if $\gamma_{1}+\delta_{1}<0$. When both SW and TW are supercritical then at most one equilibrium solution is stable. At TakensBogdanov bifurcation point we get both TW and SW. By deriving one-dimensional Ginzburg-Landau equations with complex coefficients, namely, (116) and (117), we have shown the existence of Benjamin-Feir-type of instability for both TW and SW. Near the Takens-Bogdanov bifurcation point the conducting state becomes unstable against both stationary and oscillatory mode, that is, the real parts of two eigenvalues pass through zero simultaneously. This violates the assumption made for deriving amplitude equations (60) and (98). Instead a new equation, which is second order in time, has to be used near the Takens-Bogdanov bifurcation point. 


\section{References}

[1] P. H. Roberts, D. E. Loper, and M. F. Roberts, "Convective instability of a mushy layer - I: uniform permeability," Geophysical and Astrophysical Fluid Dynamics, vol. 97, no. 2, pp. 97134, 2003.

[2] S. Chandrasekhar, Hydrodynamic and Hydromagnetic Stability, Dover, 1961.

[3] M. R. E. Proctor and N. O. Weiss, "Magnetoconvection," Reports on Progress in Physics, vol. 45, no. 11, pp. 1317-1379, 1982.

[4] S. G. Tagare, "Nonlinear stationary magnetoconvection in a rotating fluid," Journal of Plasma Physics, vol. 58, no. 3, pp. 395-408, 1997.

[5] S. G. Tagare and Y. Rameshwar, "Magnetoconvection in rotating stars," Astrophysics and Space Science, vol. 284, no. 3, pp. 983-999, 2003.

[6] P. H. Roberts and C. A. Jones, "The onset of magnetoconvection at large pradtl number in a rotating layer I. Finite magnetic diffusion," Geophysical and Astrophysical Fluid Dynamics, vol. 92, no. 3-4, pp. 289-325, 2000.

[7] R. C. Kloosterziel and G. F. Carnevale, "Closed-form linear stability conditions for magneto-convection," Journal of Fluid Mechanics, no. 490, pp. 333-344, 2003.

[8] H. R. Brand, P. C. Hohenberg, and V. Steinberg, "Codimension-2 bifurcations for convection in binary fluid mixtures," Physical Review A, vol. 30, no. 5, pp. 2548-2561, 1984.

[9] E. Palm, J. E. Weber, and O. Kvernvold, "On steady convection in a porous medium," The Journal of Fluid Mechanics, vol. 64, pp. 153-161, 1972.

[10] H. Brand and V. Steinberg, "Convective instabilities in binary mixtures in a porous medium," Physica A, vol. 119, no. 1-2, pp. 327-338, 1983.

[11] H. Brand and V. Steinberg, "Nonlinear effects in the convective instability of a binary mixture in a porous medium near threshold," Physics Letters A, vol. 93, no. 7, pp. 333-336, 1983.

[12] H. R. Brand, P. C. Hohenberg, and V. Steinberg, "Amplitude equation near a polycritical point for the convective instability of a binary fluid mixture in a porous medium," Physical Review A, vol. 27, no. 1, pp. 591-593, 1983.

[13] E. R. Lapwood, "Convection of a fluid in a porous medium," Proceedings of the Cambridge Philosophical Society, vol. 44, pp. 508-521, 1948.

[14] S. G. Tagare and A. B. Babu, "Nonlinear convection in a sparsely packed porous medium due to compositional and thermal buoyancy," Journal of Porous Media, vol. 10, no. 8, pp. 823-839, 2007.

[15] S. G. Tagare, A. B. Babu, and Y. Rameshwar, "Rayleigh-Benard convection in rotating fluids," International Journal of Heat and Mass Transfer, vol. 51, no. 5-6, pp. 1168-1178, 2008.

[16] N. Rudraiah, "Linear and non-linear magnetoconvection in a porous medium," Proceedings of the Indian Academy of Sciences (Mathematical Sciences), vol. 93, no. 2-3, pp. 117-135, 1984.

[17] N. Rudraiah and D. Vortmeyer, "Stability of finite-amplitude and overstable convection of a conducting fluid through fixed porous bed," Wärme - und Stoffübertragung, vol. 11, no. 4, pp. 241-254, 1978.

[18] R. C. Givler and S. A. Altobelli, "Determination of the effective viscosity for the Brinkman-Forchheimer flow model," Journal of Fluid Mechanics, vol. 258, pp. 355-370, 1994.

[19] A. C. Newell and J. A. Whitehead, "Finite bandwidth, finite amplitude convection," Journal of Fluid Mechanics, vol. 38, no. 2, pp. 279-303, 1969.
[20] P. C. Matthews and A. M. Rucklidge, "Travelling and standing waves in magnetoconvection," Proceedings of the Royal Society A, vol. 441, no. 1913, pp. 649-658, 1993.

[21] H. R. Brand, P. S. Lomdahl, and A. C. Newell, "Benjamin-Feir turbulence in convective binary fluid mixtures," Physica D, vol. 23, no. 1-3, pp. 345-361, 1986.

[22] V. Steinberg and H. R. Brand, "Crossover from critical to tricritical behavior in a nonequilibrium system: the convective instability in a binary fluid mixture," Physical Review A, vol. 30, no. 6, pp. 3366-3368, 1984.

[23] E. Knobloch and J. De Luca, "Amplitude equations for travelling wave convection," Nonlinearity, vol. 3, no. 4, pp. 975-980, 1990.

[24] P. Coullet, S. Fauve, and E. Tirapegui, "Large scale instability of non-linear standing waves," Journal de Physique. Lettres, vol. 46, no. 17, pp. L787-L791, 1985.

[25] A. C. Newell, "Envelope equations," in Lectures in Applied Mathematics, A. C. Newell, Ed., pp. 157-163, American Mathematical Society, Providence, RI, USA, 1974. 

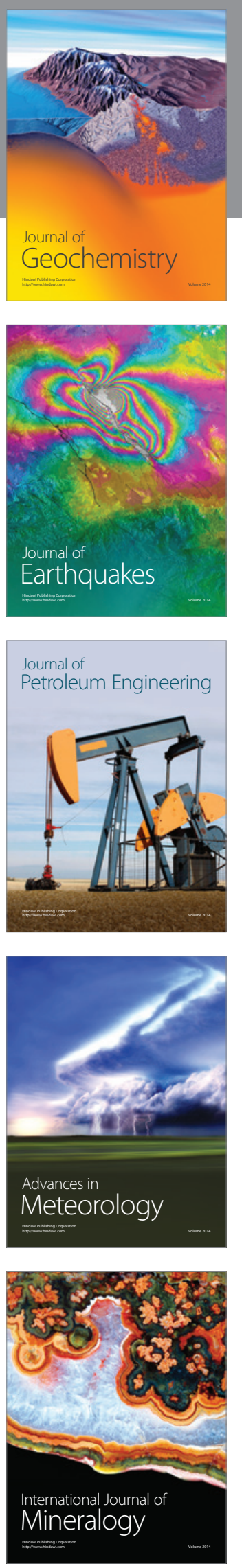
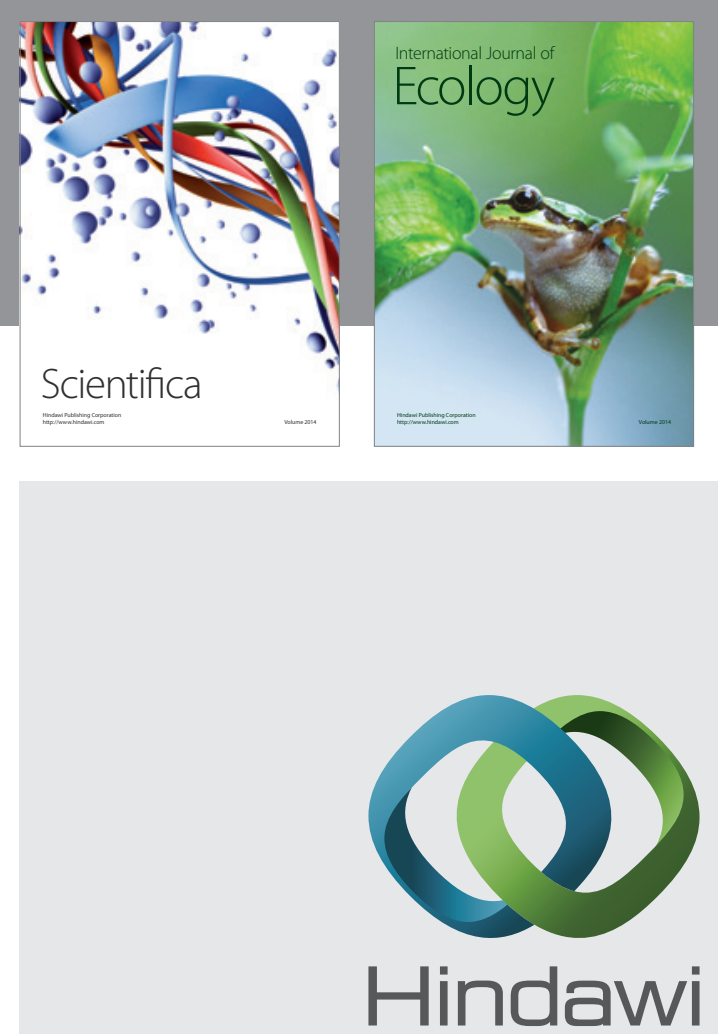

Submit your manuscripts at http://www.hindawi.com
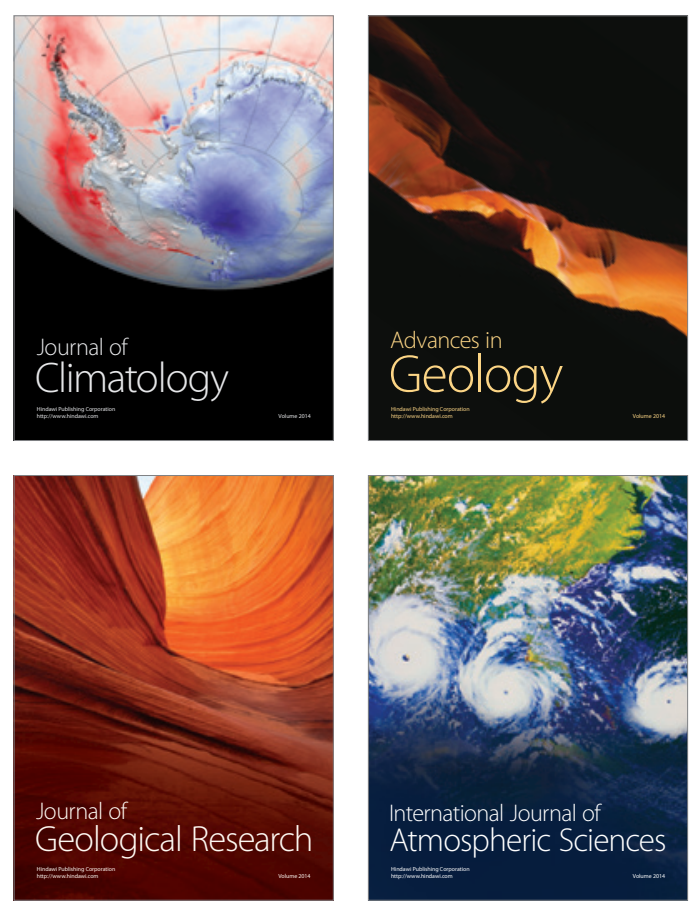
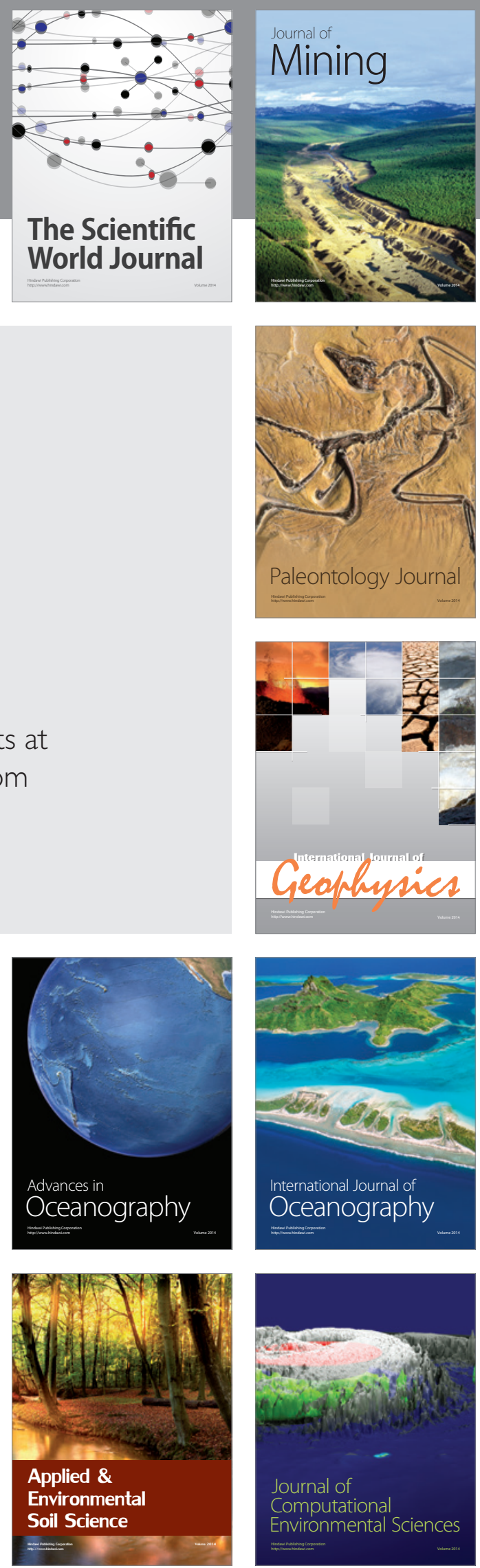